Document downloaded from:

http://hdl.handle.net/10251/46166

This paper must be cited as:

Museros Romero, P.; Moliner, E.; Martinez-Rodrigo, M. (2013). Free vibrations of simplysupported beam bridges under moving loads: Maximum resonance, cancellation and resonant vertical acceleration. Journal of Sound and Vibration. 332(2):326-345. doi:10.1016/j.jsv.2012.08.008.

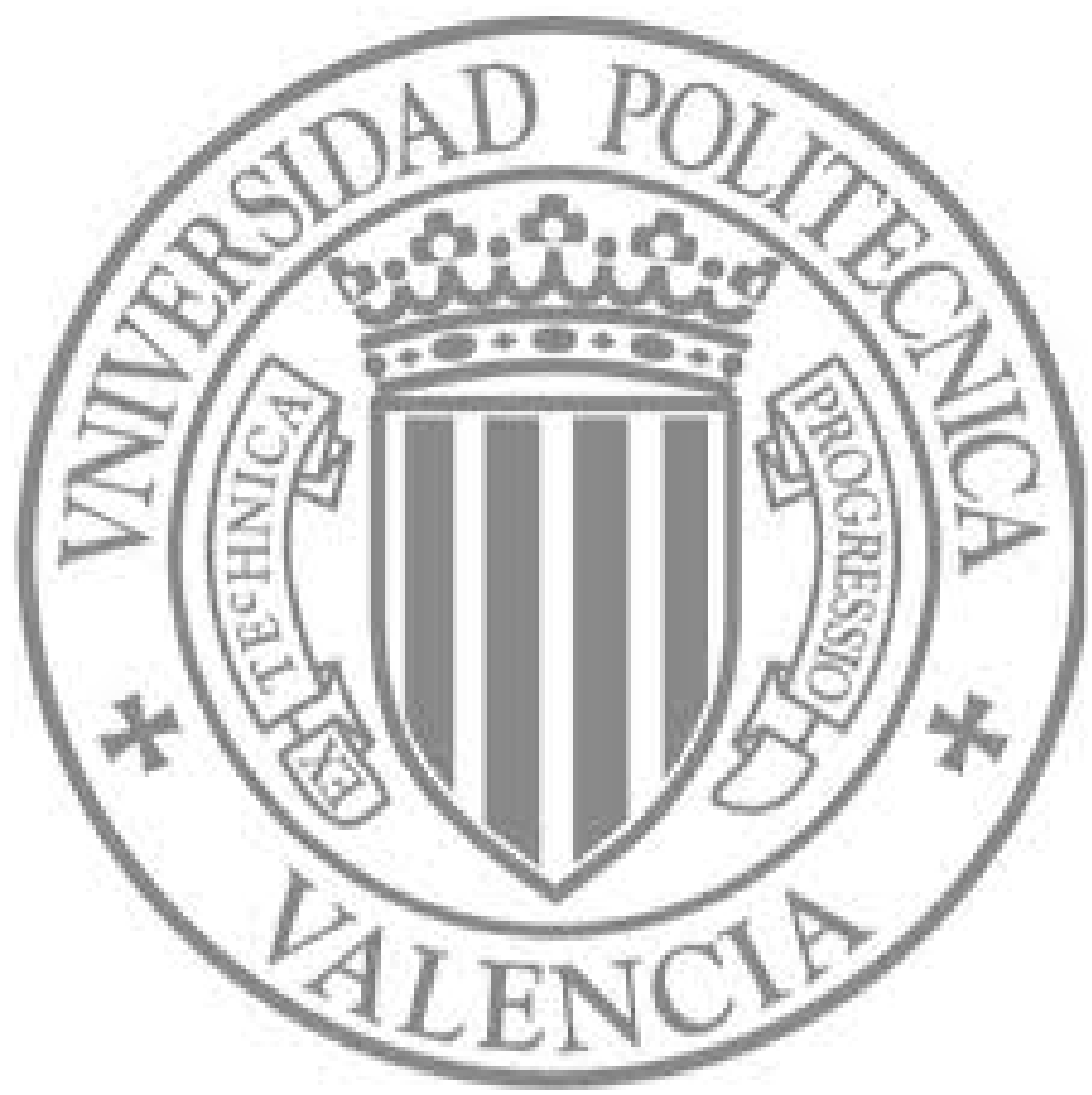

The final publication is available at

http://dx.doi.org/10.1016/j.jsv.2012.08.008

Copyright Elsevier 


\title{
FREE VIBRATIONS OF SIMPLY-SUPPORTED BEAM BRIDGES UNDER MOVING LOADS: MAXIMUM RESONANCE, CANCELLATION AND RESONANT VERTICAL ACCELERATION
}

\author{
P. Museros ${ }^{*_{a}}$, E. Moliner ${ }^{\mathrm{b}}$, M.D. Martínez-Rodrigo ${ }^{\mathrm{b}}$ \\ ${ }^{a}$ Universidad Politécnica de Valencia, Departamento de Mecánica de Medios Continuos y Teoría de \\ Estructuras, 46022 Valencia, Spain

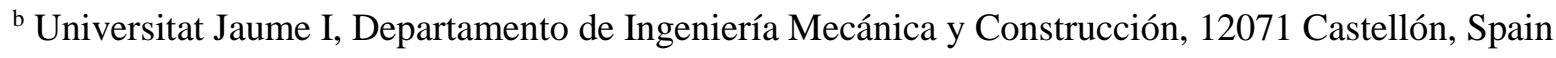

\begin{abstract}
The advent of high-speed railways has raised many concerns regarding the behaviour of bridges. Particularly, the analysis of the free vibrations generated by each load is of great interest because they can possibly accumulate and create resonance phenomena. Regarding simply supported beams, earlier contributions showed that the free vibrations created by a single moving force are of maximum or zero amplitude (cancellation) for certain speeds. In the present paper new closedform expressions are given for the cancellation speeds of a generic mode, as well as for the most representative points of maximum amplitude. Similar new results are provided for elastically supported beams as well. A simpler, closed-form approximate expression of the cancellation condition for an elastically supported beam is also derived from the analysis of a single passing load; this approximate formula is in good agreement with the exact results. Knowing a priori the speeds of maximum free vibrations or cancellation is of great interest for experimental tests on bridges, particularly as regards the evaluation of amplitude-dependent magnitudes such as structural damping. Regarding the resonance phenomena, if the resonance speeds coincide with either a maximum free vibration or a cancellation speed, then a maximum resonance or a cancellation of resonance will occur. The most relevant cases thereof have been investigated, and new expressions which allow predicting them for a generic mode are given. Finally, a new approximate formula is proposed for estimating the maximum acceleration of simply supported bridges caused by resonances of the fundamental mode. After extensive numerical testing, the formula has proved to be a useful tool for a first assessment of simply supported bridges according to building codes such as Eurocodes.
\end{abstract}

Keywords: Cancellation, resonance, moving loads, railway bridges, vertical acceleration, High-Speed trains, simply-supported beams.

\footnotetext{
* Corresponding author. Phone \#: +34-958246141, Fax \#: +34-958249959, e-mail address: pmuseros@ ugr.es
} 


\section{INTRODUCTION}

Over the last decades moving load problems have drawn the attention of many researchers from different countries, stimulated in many cases by engineering concerns arisen from the construction of new highspeed railway lines. The technical literature has increased accordingly with new theoretical developments and interesting practical applications in different fields: fast trains moving on embankment, or travelling through tunnels — which creates also aerodynamic problems — or, very often, high-speed trains crossing bridges of different kinds: single-track ones, double-track ones, simply supported or continuous ones, arches, suspended bridges, etc.

The theoretical bases of problems related to vehicles crossing over bridges are well known. Many contributions have enriched this discipline over the past two centuries: a historic review of the successive developments can be found in the works of Frýba [1], Weaver et al. [2], Michaltsos [3] or Savin [4]. Due to the advent of high speeds, the problem of resonance, particularly in simply supported bridges, became one of the most active fields of research. From a theoretical perspective, researchers have approached this problem in different ways. Very often the common starting point has been the closed-form solution obtained by Kryloff [5] for the simple beam subjected to a constant travelling force. For simply supported bridges, the Kryloff solution has indeed become the most popular one, being further investigated by many authors [6]-[12]. Besides, it has been adopted as a theoretical basis by a number of regulations as, for instance, Eurocode 1 [13].

When a simple beam is subject to trains of equidistant loads, the time interval between the pass of consecutive loads is one of the governing parameters, and peak responses are attained when this interval is equal to (or an integer multiple of) one of the natural periods of the beam. It appears that Bolotin [14] and Fryba [1] were the first ones to publish these important conclusions. More recently, Klasztorny and Langer [15] analysed the resonance problem considering a variety of periodic load models travelling over a simply supported beam. Using a nondimensional formulation, they explained most of the characteristics of the resonance phenomenon, as well as the differences between the results obtained with the different models, particularly for metallic railway bridges.

An interesting contribution about resonance problems in simple bridges was later presented by Yang et al [16]. In this reference the resonance conditions were derived for a train consisting of a series of equidistant groups of loads, as it is the case of most modern high-speed trains. The authors also derived the cancellation conditions for such kind of trains: for certain ratios between span length $L$ and characteristic (repetitive) load distance $d$, the expected resonant response does not take place. They linked this phenomenon to the suppression of the accumulated vibrations generated by the series of moving loads.

A few years after, Frýba proposed simplified formulas for computing the bridge response (deflection, 
bending moment and acceleration) when resonance of the first mode of a simply supported beam takes place [17]. He considered an excitation composed by a series of concentrated, equidistant loads which excite the first resonance case, i.e. the case when there is just one cycle of oscillation of the beam between the pass of consecutive loads.

Almost simultaneously, Savin analysed thoroughly the importance of free vibrations and how they combine to give the highest response of the beam [4]. He furnished approximate expressions to compute the displacement and vertical acceleration from a passing train; these expressions are based on a spectrum of the train which is able to provide the response at any circulating speed. The spectrum introduced by Savin is virtually identical to the so-called dynamic signature introduced by the LIR (Residual Influence Line) method developed by SNCF (Societé Nationale des Chemins de Fer) in the framework of the ERRI D-214 Committee [18]. This method is coincident with the approach adopted by Savin in the sense that the bridge response is separated into three terms: a constant which is a sole function of the bridge, a second term called influence line or dynamic amplification factor which evaluates the amplitude of the free vibrations produced by a single load, and a third term (dynamic signature) which estimates how the effects of the different loads of the train combine, for a given value of structural damping.

Also, a similar factorisation of the bridge response is achieved by another procedure developed within the work of the ERRI D-214 Committee: the Decomposition of Excitation at Resonance method (DER). The DER method is based in a Fourier decomposition of the excitation which retains only the term closest to the resonant frequency of the fundamental bending mode; therefore, it is based in a principle different from the ones followed by Savin or the LIR method, but the prediction of the response obtained for the resonant speeds is very similar [18]. The DER and LIR methods introduced a definition of the dynamic signature of a train (equivalent for undamped structures) which is of great importance for interoperability specifications, and both methods are at the basis of the present regulations prescribed by Eurocode 1 .

Savin also clarified in [4] the phenomenon of cancellation by explaining that the dynamic amplitude of the free vibrations of each single load vanishes for certain speeds, which suppresses the resonance phenomenon for certain $L / d$ ratios. In the present paper further insight into the reasons of cancellation for the different bending modes will be given, and the conclusions will be also applied to beams resting on elastic supports. The resonant behaviour of this kind of structures was previously analysed by Yang et al [19] using an approximate expression of the first bending mode. An analytic approach will be used here, and some results will be given for usual values of supports stiffness. Also, the phenomenon of maximum resonance will be explained on the basis of the maximum free vibrations both for simply supported and elastically supported bridges.

Aside from the aforementioned relevant contributions to the analysis of resonance phenomena in simple beams, other authors refer to different kinds of resonant behaviours. Xia et al. [20] investigated the 
rhythmic vibrations created by the pass of a sprung vehicle over consecutive spans of a bridge, or by the sway movements induced by wheel/rail irregularities. Regarding suspended bridges, $\mathrm{Xu}$ et al. [21] have suggested that it is more likely that moving vehicles excite higher mode resonances in these structures than in simply supported bridges.

Among the consequences of resonant behaviour, it is of special interest to analyse the vertical acceleration experienced by simple beams; as it is known, this magnitude constitutes a determinant factor for the design of such structures in high-speed railway lines. Apart from Savin [4] and the ERRI [18], the acceleration has been investigated by Museros and Alarcón [22], who adopted a combined analytical/numerical approach in order to investigate the influence of the second bending mode. Also, Yau and Yang [23] pursued similar objectives following a more refined analytic procedure. In the present paper, a new and rather simple formula will be proposed for estimating the maximum value of the acceleration caused by resonances of the fundamental mode. This formula is based on the analysis of the free vibrations, which are subsequently combined in the possible resonance scenarios. The formula can tackle with any number of cycles of oscillation between the pass of consecutive loads, and it takes also account of the out-of-phase contributions of different axles belonging to a same bogie. As it will be shown, it can be a useful tool for a first assessment of simply supported bridges according to building codes such as the Eurocode.

Finally, it should be remarked that the interest in free vibrations is not limited to resonance phenomena. When dynamic tests are carried out on road or railway bridges, a typical source of excitation can be a vehicle travelling over the structure at different speeds. In such circumstances it is convenient to maximise the vibrations in order to have a large amplitude signal. Knowing a priori the speeds which cause maximum free vibrations can help one to obtain good measurements and, therefore, good estimates of the dynamic properties of the bridge — as, for instance, structural damping.

\section{FREE VIBRATIONS OF BEAMS UNDER A SINGLE MOVING LOAD}

\subsection{Simply-supported beams}

The equation of motion of a simply supported (S-S) uniform beam traversed by a constant-valued load moving at constant speed, where damping is neglected as well as shear deformation and rotary inertia effects, is well known and its solution is available from many authors already mentioned in the previous section. If this solution $v=v(x, t)$ is expressed as a combination of eigenfunctions normalised so as to have unitary maximum amplitude one has 
$v(x, t)=\sum_{n=1}^{\infty} \xi_{n}(t) \sin \frac{n \pi x}{L}$,

and the $\mathrm{n} t h$ modal amplitude $\xi_{n}$ is obtained as a function of time during the forced vibration period $(0 \leq t \leq L / V)$ as follows:

$\begin{array}{ll}\xi_{n}(t)=-\frac{2 P}{m L \omega_{n}^{2}} \frac{1}{1-K_{n}^{2}}\left[\sin \left(K_{n} \omega_{n} t\right)-K_{n} \sin \left(\omega_{n} t\right)\right], & 0 \leq t \leq L / V, \quad K_{n} \neq 1, \\ \xi_{n}(t)=-\frac{2 P}{m L \omega_{n}^{2}} \frac{1}{2}\left[\sin \left(\omega_{n} t\right)-\omega_{n} t \cos \left(\omega_{n} t\right)\right], & 0 \leq t \leq L / V, \quad K_{n}=1 .\end{array}$

In Eqs. (1) and (2) $L$ is the length of the beam, $P$ is the concentrated moving load and $V$ its constant speed (see Fig. 1a). The mechanical characteristics of the beam are its linear mass $m$ and bending stiffness $E I_{z}$, and $\omega_{n}$ is the $n$th circular frequency of the beam:

$\omega_{n}=(n \pi / L)^{2} \sqrt{E I_{z} / m}$.

Following the tradition of the International Union of Railways (UIC), the nondimensional speed $K_{n}$ is defined as the ratio of the forcing and natural frequencies

$K_{n}=\frac{\Omega_{n}}{\omega_{n}}=\frac{n \pi V}{\omega_{n} L}=\frac{V L}{n \pi} \sqrt{\frac{m}{E I_{z}}}=\frac{1}{n} K$,

where $K=K_{1}$ is the nondimensional speed for the first mode. In what follows subscript "1" will be dropped for magnitudes relative to the fundamental mode provided that it does not lead to confusion. The so-called static solution is also recognised in Eqs. (2):

$\xi_{n, s t}=\frac{2 P}{m L \omega_{n}^{2}}$.

After the load has passed the beam, the $n$th mode experiences subsequently the so-called free vibrations, which are governed by

$\ddot{\xi}_{n}(t)+\omega_{n}^{2} \xi_{n}(t)=0, \quad t>L / V$.

For practical purposes the discussion will be restricted to the case $K_{n}<1$, which is the one of interest for real bridges as it will be shown. Therefore, attention will be focussed in Eq. (2a). The free vibration for $K_{n} \neq 1$ is the solution of Eq. (6) with initial conditions as provided by Eq. (2a) particularised at $t=L / V$. It is straightforward to prove that the free vibration can be written as a single sine or cosine function; the amplitude of such function, divided by the static value given by Eq. (5), is equal to 
$R_{n}=\frac{K_{n} \sqrt{2}}{1-K_{n}^{2}} \sqrt{1-\cos n \pi \cdot \cos \frac{n \pi}{K_{n}}}$.

Therefore, Eq. (7) is of great interest and it may be referred to as the normalised amplitude of the free vibrations. Fig. 2 shows the variation of $R_{n}$ with $K_{n}$ for the first mode. As it had been shown by Pesterev et al [12], a global maximum and a number of local maxima alternate with points of zero amplitude: these are the phenomena of maximum dynamic response and cancellation, as regards the free vibration response of the beam to a moving load. The evolution of $R_{n}$ can be shown to be very similar also for higher modes.

\subsubsection{Condition for maximum dynamic response}

In what follows indexes $i$ and $j$ will be considered positive integers. The points of maximum free vibrations observed in Fig. 2 are obtained from equating to zero the derivative of Eq. (7). The mathematical manipulations become simpler if even and uneven values of the mode number $n$ are treated separately. In doing so, after some straightforward operations the following novel expressions are obtained:

$$
\begin{array}{ll}
\frac{\partial R_{n}}{\partial K_{n}}=0 \Leftrightarrow \tan \frac{n \pi}{2 K_{n}}=\frac{2 K_{n}\left(1+K_{n}^{2}\right)}{n \pi\left(K_{n}^{2}-1\right)}, & n=2 j-1, \quad j \geq 1, \\
\frac{\partial R_{n}}{\partial K_{n}}=0 \Leftrightarrow \tan \frac{n \pi}{2 K_{n}}=\frac{n \pi\left(1-K_{n}^{2}\right)}{2 K_{n}\left(1+K_{n}^{2}\right)}, \quad n=2 j, \quad j \geq 1
\end{array}
$$

The solutions to Eqs. (8) are to be sought by numerical procedures. These solutions will be named $K_{n i}^{m}$, where superscript $m$ indicates a value of $K_{n}$ associated to a maximum of the free vibrations, and subscript $i$ designates the $i$ th root of Eq. (8) for the $n$th mode. Value $i=1$ will correspond to the root of higher speed $K_{n}$, which is the global maximum; $i=2$ will be the following root in decreasing order of speed and so on (see Fig. 2).

Fig. 2 shows that the maximum free vibrations after the pass of a load depend on how elevated is the maximum nondimensional speed. Because in engineering applications the fastest moving loads are found in high-speed railway lines and Maglev lines, one can take real data from existing railway bridges in order to obtain an approximation for the maximum expected values of $K$. It is remarked that the maximum nondimensional speed corresponds always to the first mode, according to Eq. (4). Since high-speed bridges are usually stiff, a minimum frequency for each span length can be estimated as the average of the upper and lower limits in Fig. 3, which is taken from reference [24]. Simultaneously, an operating speed of $500 \mathrm{~km} / \mathrm{h}$ can be considered as an upper limit for conventional high-speed trains and a reasonable value for nowadays Maglev trains. By combining both assumptions an estimation of the maximum value of $K$ is obtained, as shown in Fig. 4. For the shortest spans, $K$ takes its largest values, which are about 0.55. 
Therefore, for practical purposes it is of interest to locate the maxima excluding the ones above $K=0.55$. Fig. 2 also shows that the global maximum is not expected to be attained in practical engineering applications; this maximum would correspond to the "first" root $K_{11}^{m}=0.7314$ (first mode), $K_{21}^{m}=0.8883$ (second mode) and $K_{31}^{m}=0.9422$ (third mode).

Of special interest is the contribution of the first mode, as it has been noted by several authors $[1,4,10$, $12,16]$. The three highest local maxima for this mode, excluding $K_{11}^{m}$, are gathered in Table 1 in decreasing order of $K$. They are shown along with the values corresponding to beams on elastic supports (see section 2.2); therefore, they correspond to the row $\kappa=0$, which means zero flexibility of the supports. In Table 1 a value $\hat{K}_{1}^{m}$ is also included which corresponds to the condition defined by $R_{1}\left(\hat{K}_{1}^{m}\right)=R_{1}\left(K_{12}^{m}\right)=R_{1}(0.2576)$ (see Fig. 2). If the expected maximum speed of the moving loads is such that $K>\hat{K}_{1}^{m}=0.3858$, then the maximum free vibration will be attained at the maximum circulating speed. For the sake of completeness, Table 1 includes also analogous results for the second mode.

\subsubsection{Condition for cancellation}

Fig. 2 shows that the amplitude of the free vibrations vanishes for certain values of $K_{n}$, as it has been anticipated by Savin [4] and Pesterev et al. [12]. This is a curious consequence of the addition of the particular and homogeneous components of the forced vibrations given by Eq. (2a): since the initial conditions of the differential equation leading to Eqs. (2) are homogeneous for all modes

$$
\xi_{n}(0)=\dot{\xi}_{n}(0)=0
$$

then the homogeneous and particular components of the solution take zero initial values and equal (but opposite sign) initial slopes. This is true regardless of the value of $K_{n}$. For values of $K_{n} \neq 1$, the particular solution will experiment $n$ half-cycles during the forced vibration phase, also regardless of $K_{n}$. If the speed is such that the homogeneous solution performs a number of half-cycles equal to $n \pm 2 i$, where $i \geq 1$, then the final values of both components at $t=L / V$ will be zero, and their final slopes will be again opposite and cancel each other. This is a new, simple and rather illustrative interpretation of the phenomenon of cancellation.

Fig. 5 shows this kind of phenomenon for the first mode. A nondimensional time $\tau$ has been used such that $\tau=1$ when the load leaves the beam. Plot 5a shows how the particular and homogeneous solutions combine to generate the free vibration in a general case $(K=0.375)$. Plot $5 \mathrm{~b}$ shows that the free vibrations cancel out completely when the homogeneous solution performs three half-cycles $(K=1 / 3)$.

For the first mode, the cancellation phenomenon takes place for any odd number of half-cycles except for $K=1$. In general, cancellation cannot occur for any mode if $K_{n}=1$. This is the so-called critical case, when the solution of the forced vibration is given by Eq. (2b). It can be proved easily that Eq. (2b) is never zero 
for $t=L / V$, while its derivative is zero at this precise instant; therefore the existence of nonzero amplitude free vibrations is proved.

In summary, this interpretation of cancellation can be expressed analytically by the following, simple Eq. (11), which is new and valid for a generic mode: the number of half-cycles performed by the homogeneous solution while the load is on the beam is

$N_{n}=\frac{L / V}{T_{n} / 2}=n \frac{\omega_{n} L}{n \pi V}=\frac{n}{K_{n}}, \quad T_{n}=\frac{2 \pi}{\omega_{n}}$,

and cancellation will occur for the $n$th mode providing that

$N_{n}=n \pm 2 i>0, \quad i \geq 1 \quad \Leftrightarrow \quad K_{n i}^{c}=\frac{n}{n \pm 2 i}>0, \quad i \geq 1$

where superscript $c$ indicates cancellation and subscript $i$ designates the number of the cancellation phenomenon. The first cancellations for the first four modes are listed in Table 2. They correspond to the largest possible values of $K_{n}$. Modes 3 and 4 present negative values of index $i$; this entails that the minus sign in Eq. (11) has been used, and the homogeneous solution performs less cycles than the particular one. In Fig. 6 the first two possible cancellations for the first three modes are shown.

Regarding the influence of structural damping — which had been disregarded in this paper so far-Savin [4] showed that small damping alters but slightly the speeds of cancellation for the first two modes, while the associated amplitude of free vibrations reduces almost to zero but not completely. Therefore the essential features of the phenomenon are not altered significantly in damped structures.

When the response of one of the modes cancel, the total response of the beam is not zero because clearly the rest of the modes may still contribute. But as it was mentioned before, the a priori knowledge of the speeds of maximum free vibration and cancellation per mode can be of practical interest, particularly because of the two following facts:

1) For the experimental measurement of bridge properties engineers often carry out field tests based on the analysis of the oscillations caused by the pass of a single truck or locomotive. Some of the objectives of such tests it to measure important modal parameters as, for instance, modal damping. Therefore it could be of much help to know a priori what speeds of the proof load vehicles may produce a high level of free vibrations of a particular mode after leaving the structure, and which ones are susceptible to cancel the free vibrations of such mode. The test engineers may estimate the frequencies of simply supported bridges from an ambient vibration test (or using a vehicle passing at random speeds), and then by subsequent application of the maximum free vibration predicted in 
sections 2.1.1 and 2.2.1 they will be able to maximize the vibration amplitude.

2) According to the regulations existing in most countries, during the design of a simply supported bridge different types of load trains are used for the purpose of dynamic analyses. Some of them will produce resonance of the first mode, and it is most likely that this will be the predominant phenomenon. For instance, the ten trains comprised in the HSLM-A model from Eurocode [13] are designed purposely so as to produce such effect. Therefore, it is of interest for the engineers to know when resonance of the first mode will be maximum and when it could cancel, because this information can be used in order to check whether the numerical analysis carried out with a computer or spreadsheet are correct. On the basis of the results presented in section 2, the phenomena of maximum resonance and cancellation of resonance are analyzed in sections 3 and 4 .

\subsubsection{Effects of vehicle-bridge interaction}

Evaluating the difference between the results obtained from constant moving load models and vehicle-bridge interaction (VBI) models is always a subject of interest among scientists and engineers. While this interest is undeniable, it should also be emphasized that understanding the key factors and predominant phenomena in constant moving loads models has an interest per se, particularly because such models are widely used for design purposes according to the regulations existing in most modern countries, as it was mentioned previously.

As regards the phenomena of maximum free vibration and cancellation, VBI effects play an increasing role when two factors contribute simultaneously: (i) the frequency of the suspended masses of the vehicle approaches the natural frequencies of the bridge (i.e. the frequency ratios are close to unity) and (ii) the suspended mass is relatively large compared to the bridge mass (the mass ratio takes large values). The influence of these two nondimensional ratios has been analyzed previously by the authors: since they do affect the amplitude of the free vibrations, they also affect how vibrations may accumulate and create resonance phenomena; therefore, it is seen that for frequency ratios close to unity and higher mass ratios the VBI effects tend to reduce the peak resonant accelerations $[25,26]$.

There are two types of suspended masses in railway vehicles, the sprung ones (masses of the car-bodies) and the semi-sprung ones (masses of the bogies). The maximum mass of the car-bodies is elevated: it can reach some $35000 \mathrm{~kg}$ for conventional passenger trains, but conversely their natural frequencies are very low (not far from $1 \mathrm{~Hz}$ ). For this reason, the frequency ratios with the majority of S-S bridges are always far from unity and VBI effects due to car-bodies are of little importance. Consequently, even if one takes into account the interaction between car-bodies and bridge, Eqs. (8) and (11) can generally be considered good estimates for the maximum free vibration and cancellation speeds.

Regarding the interaction with the bogies, their natural frequencies are approximately in the range [4Hz, 
$8 \mathrm{~Hz}$, but their maximum mass value is low compared to car-bodies: about $3000 \mathrm{~kg}$ for passenger cars and 5000 for locomotives. Therefore, for low-mass bridges whose first bending frequency is similar to the semi-sprung mass frequency, the lowest speeds of cancellation and maximum free vibrations can be altered; this effect is felt particularly at lower speeds $(K<0.15 \div 0.2)$, because bridge and vehicle interact during more cycles of oscillation. Nevertheless, low-mass S-S structures are becoming less common in modern railway lines, precisely because of their tendency to experience too strong vibrations. In modern bridges the mass ratio of the bogies to the bridge will usually be low, and the maximum free vibration and cancellation speeds will still be well approximated by Eqs. (8) and (11). However, when the ratio between the bogie frequency and the bridge fundamental frequency is close to unity the amplitudes of the free vibrations decrease with respect to Fig. 2; in case of resonance due to the pass of multiple loads this effect may produce a reduction of the maximum vibration amplitude. Following previous results from references $[25,26]$, this phenomenon will be explored in detail in subsequent publications.

\subsection{Beams on elastic supports}

Pursuing further the problem of free vibrations, in this section the response of elastically-supported (E-S) beams under the circulation of a constant moving load is analysed. Fig. $1 \mathrm{~b}$ shows the planar model of a simple bridge leaning on identical sets of elastic bearings. In the next derivations the following is assumed: (i) in the range of expected strains the vertical stiffness of each elastic support is constant and equal to $K_{v}$; (ii) the mass of the bearings is negligible when compared to the beam total mass; (iii) structural damping, shear deformation and rotary inertia of the beam are neglected.

The transverse deflection of the beam is again expressed as a linear combination of a series of normal modes $\phi_{n}(x)$, normalised for maximum unitary amplitude

$$
v(x, t)=\sum_{n=1}^{\infty} \xi_{n}(t) \phi_{n}(x)
$$

Exact frequency equations and mode shapes of uniform beams with elastic supports may be found in [27, 28]. In the particular case of study the frequency equation is given by

$$
\left(\frac{\pi^{3}}{\kappa}\right)^{2}+\frac{\pi^{3}}{\kappa} \lambda^{3} \frac{(\sinh (\lambda) \cos (\lambda)-\cosh (\lambda) \sin (\lambda))}{\sin (\lambda) \sinh (\lambda)}+\lambda^{6} \frac{(1-\cos (\lambda) \cosh (\lambda))}{2 \sin (\lambda) \sinh (\lambda)}=0,
$$

being $\kappa=E I_{z} \pi^{3} /\left(K_{v} L^{3}\right)$ the ratio of the flexural rigidity of the beam to the stiffness of the elastic bearings. As it is known, the roots of Eq. (13), $\lambda_{n}=\lambda_{n}(\kappa)$, relate to the circular frequencies of the E-S and S-S beams as follows: 
$\bar{\omega}_{n}=\left(\frac{\lambda_{n}}{L}\right)^{2} \sqrt{\frac{E I_{z}}{m}} \Leftrightarrow \frac{\bar{\omega}_{n}}{\omega_{n}}=\left(\frac{\lambda_{n}(\kappa)}{n \pi}\right)^{2}$.

The magnitudes of speed and frequency obtained for the E-S beam will be identified by an overbar. They are equal to the ones from the $S-S$ case for $\kappa=0$ because $\lambda_{n}(\kappa=0)=n \pi$. The analytical mode shapes for each value of $\lambda_{n}$ are $[27,28]$

$\phi_{n}(x)=\frac{\psi_{n}(x)}{\operatorname{Max}\left|\psi_{n}(x)\right|}$,

where

$$
\begin{aligned}
& \psi_{n}(x)=\sin \left(\frac{\lambda_{n} x}{L}\right)+\sinh \left(\frac{\lambda_{n} x}{L}\right) \frac{\sin \left(\lambda_{n}\right)}{\sinh \left(\lambda_{n}\right)}+\gamma_{1 n}\left(\cos \left(\frac{\lambda_{n} x}{L}\right)+\cosh \left(\frac{\lambda_{n} x}{L}\right)+\gamma_{2 n} \sinh \left(\frac{\lambda_{n} x}{L}\right)\right) \text { and } \\
& \gamma_{1 n}=\frac{\sinh \left(\lambda_{n}\right)-\sin \left(\lambda_{n}\right)}{\frac{2}{\kappa}\left(\frac{\pi}{\lambda_{n}}\right)^{3} \sinh \left(\lambda_{n}\right)+\cos \left(\lambda_{n}\right)-\cosh \left(\lambda_{n}\right)}, \quad \gamma_{2 n}=\frac{\cos \left(\lambda_{n}\right)-\cosh \left(\lambda_{n}\right)}{\sinh \left(\lambda_{n}\right)} .
\end{aligned}
$$

The evolution of the $n$th modal amplitude during the forced vibration period can be obtained by standard procedures as shown by Dmitriev [29] for symmetric and antisymmetric modes separately. Alternatively, if the mode shapes as given in Eqs. (15) are used, a new and single expression for the modal response of the beam to the moving load can be derived:

$$
\begin{gathered}
\frac{\xi_{n}(t)}{\xi_{n, s t}}=\frac{-1}{\operatorname{Max}\left|\psi_{n}(x)\right|}\left[\frac{\bar{K}_{n} \sin \left(\bar{\omega}_{n} t\right)-\sin \left(\bar{K}_{n} \bar{\omega}_{n} t\right)}{\bar{K}_{n}^{2}-1}+\left(\frac{\sin \left(\lambda_{n}\right)}{\sinh \left(\lambda_{n}\right)}+\gamma_{1 n} \gamma_{2 n}\right) \frac{\sinh \left(\bar{K}_{n} \bar{\omega}_{n} t\right)-\bar{K}_{n} \sin \left(\bar{\omega}_{n} t\right)}{\bar{K}_{n}^{2}+1}+\right. \\
\left.+\gamma_{1 n} \frac{\cos \left(\bar{\omega}_{n} t\right)-\cos \left(\bar{K}_{n} \bar{\omega}_{n} t\right)}{\bar{K}_{n}^{2}-1}+\gamma_{1 n} \frac{\cosh \left(\bar{K}_{n} \bar{\omega}_{n} t\right)-\cos \left(\bar{\omega}_{n} t\right)}{\bar{K}_{n}^{2}+1}\right], \quad 0 \leq t \leq L / V,
\end{gathered}
$$

where $\xi_{n, s t}$ stands for the static solution

$$
\xi_{n, s t}=\frac{P}{M_{n} \bar{\omega}_{n}^{2}}
$$

$M_{n}$ represents the $n$th modal mass in the case of unitary amplitude normalisation of the modes, i.e

$$
M_{n}=\int_{0}^{L} m \phi_{n}(x)^{2} \mathrm{~d} x=\frac{\int_{0}^{L} m \psi_{n}(x)^{2} \mathrm{~d} x}{\left[\operatorname{Max}\left|\psi_{n}(x)\right|\right]^{2}}
$$

and the speed parameter $\bar{K}_{n}$ is defined again as the ratio between the forcing frequency of the load, 
$\bar{\Omega}_{n}=\lambda_{n} V / L$, and the E-S beam $n$th circular frequency, i.e.

$\bar{K}_{n}=\frac{\bar{\Omega}_{n}}{\bar{\omega}_{n}}=\frac{\lambda_{n} V}{\bar{\omega}_{n} L}=\frac{V L}{\lambda_{n}} \sqrt{\frac{m}{E I_{z}}}$.

According to Eq. (4), the following useful relation exists:

$$
K_{n}=\frac{\bar{K}_{n} \lambda_{n}}{n \pi}
$$

For certain purposes parameter $K_{n}$ is preferable to $\bar{K}_{n}$ because it is independent of $\kappa$. After the load leaves the beam, the response in the free vibration phase is obtained with the initial conditions $\dot{\xi}_{n}(t=L / V)$ and $\xi_{n}(t=L / V)$, as provided by Eq. (16). Closed form expressions of such initial conditions can be obtained, but they are rather involved and of little practical interest. Instead, for computation purposes it is preferable to evaluate $\dot{\xi}_{n}(t=L / V)$ and $\xi_{n}(t=L / V)$ in each case and obtain the free vibration as

$\xi_{n}(t)=\frac{\dot{\xi}_{n}(t=L / V)}{\bar{\omega}_{n}} \sin \left(\bar{\omega}_{n}\left(t-\frac{L}{V}\right)\right)+\xi_{n}(t=L / V) \cos \left(\bar{\omega}_{n}\left(t-\frac{L}{V}\right)\right), \quad t>L / V$.

Finally, the amplitude of the $n$th modal response in the free-vibration phase, normalised by the static solution, can be computed as

$$
R_{n}=\frac{1}{\xi_{n, s t}} \sqrt{\frac{\dot{\xi}_{n}(t=L / V)^{2}}{\bar{\omega}_{n}^{2}}+\xi_{n}(t=L / V)^{2}},
$$

Eq. (22) is therefore the normalised amplitude of the free vibrations of the $n$th mode of an E-S beam travelled by a constant moving load $P$. In Fig. $7 R_{n}$ has been represented for the first two modes of vibration of the E-S beam, with $\kappa$ in the range [0,0.2]; the higher limit of this interval of $\kappa$ is an approximate upper bound to the values expected in most short to medium-span railway bridges. The plot is given in terms of $K_{n}=\bar{K}_{n} \lambda_{n}(n \pi)^{-1}$. One conclusion of this investigation is that the modal responses obtained for the E-S beam follow a similar pattern as those corresponding to the S-S case: cancellation and maximum free vibration situations alternate as the speed increases, while the nondimensional flexibility $\mathrm{\kappa}$ tends to reduce the corresponding values of speed (more noticeably as the mode number increases). In the following sections the conditions for both maximum response and cancellation of the free vibration are investigated.

\subsubsection{Conditions for maximum dynamic response}

The particular values of the speed parameters $\bar{K}_{n}$ leading to a maximum of the free vibration response in the E-S case may be computed by numerical differentiation of $R_{n}$, i.e 


$$
\frac{\partial R_{n}}{\partial \bar{K}_{n}}=0 \Rightarrow \frac{\partial}{\partial \bar{K}_{n}} \sqrt{\frac{\dot{\xi}_{n}(t=L / V)^{2}}{\bar{\omega}_{n}^{2}}+\xi_{n}(t=L / V)^{2}}=0 .
$$

The numeric solutions of Eq. (23) are $\bar{K}_{n i}^{m}(\kappa)$; the corresponding true speeds $\bar{V}_{n i}^{m}(\kappa)$ are given by Eq. (19). With the purpose of using a common nondimensional basis, $\bar{K}_{n i}^{m}(\kappa)$ can be transformed by means of Eq. (20) and will be named simply $K_{n i}^{m}$ as the solutions to Eqs. (8). Table 1 gathers the values of $K_{n i}^{m}$ for $n=1,2$ and $i=2,3,4$ in the range of the supports flexibility $\kappa=[0,0.2]$. The value $\hat{K}_{n}^{m}$ corresponding to a free vibration defined by the condition $R_{n}\left(\hat{K}_{n}^{m}\right)=R_{n}\left(K_{n 2}^{m}\right)$ has been included as well.

\subsubsection{Conditions for cancellation}

Maxima of $R_{n}$ alternate with cancellation situations as in S-S beams. For certain values of $\bar{K}_{n}$, the homogeneous and particular solutions of the beam forced response cancel each other when the load exits the beam, leading to a zero amplitude free vibration of the structure. From Eq. (16) these homogeneous and particular solutions of the forced response may be separated, but the mathematical expressions of cancellation are too involved as to produce simple and useful closed-form formulas. Consequently, as it was done with the maxima, the speeds leading to cancellation are obtained numerically as the roots of the following equation:

$$
R_{n}=0 \Rightarrow \sqrt{\frac{\dot{\xi}_{n}(t=L / V)^{2}}{\bar{\omega}_{n}^{2}}+\xi_{n}(t=L / V)^{2}}=0 .
$$

The solutions to Eq. (24) are $\bar{K}_{n i}^{c}(\kappa)$, and the associated true speeds $\bar{V}_{n i}^{c}(\kappa)$. Following a transformation by application of Eq. (20), $\bar{K}_{n i}^{c}(\kappa)$ will be designated $K_{n i}^{c}$ as in Eq. (11). Values corresponding to the first four cancellations of the first two modes are shown in Table 1.

\subsubsection{Approximate expression for cancellation speeds of the first bending mode}

In addition to Eq. (24), an approximate expression can be found to obtain the speeds leading to cancellation of the first bending mode of an E-S beam. This simplified equation is sought by considering an approximate deformed shape of the first vibration mode of the E-S beam, previously introduced by Yang et al [19]:

$\Phi(x)=\sin \frac{\pi x}{L}+\kappa$

Eq. (25) is the superposition of the first bending mode shape of the S-S beam and the symmetric mode shape of a rigid beam supported by elastic springs. The approximate deformed shape $v(x, t)$ of the E-S beam, accounting only for the first modal contribution, is expressed as

$$
v(x, t)=\xi(t)\left(\sin \frac{\pi x}{L}+\kappa\right) .
$$


By substituting Eq. (26) into the differential equation of motion of a simply supported, undamped beam, multiplying by Eq. (25) and integrating along the length $L$ of the beam, one obtains a differential equation for the forced vibrations:

$$
\ddot{\xi}(t)+\tilde{\omega}^{2} \xi(t)=-\frac{2}{m L} P\left(1+\frac{8 \kappa}{\pi}+2 \kappa^{2}\right)^{-1}\left(\sin \left(\frac{\pi V t}{L}\right)+\kappa\right), \quad 0 \leq t \leq L / V
$$

where $\tilde{\omega}$ is the approximate frequency of vibration of the beam with elastic supports,

$$
\tilde{\omega}=\left(\frac{\pi}{L}\right)^{2} \sqrt{\frac{E I}{m}} \sqrt{\frac{\pi+4 \kappa}{\pi+8 \kappa+2 \pi \kappa^{2}}}
$$

$\tilde{K}$ is equal to the speed parameter $S$ from reference [19]:

$$
\tilde{K}=\frac{\pi V}{\tilde{\omega} L}
$$

The generalised coordinate $\xi(t)$ is solved from Eq. (27) considering homogeneous initial conditions, as

$$
\xi(t)=-\frac{2 P L^{3}}{E I \pi^{4}\left(1+\frac{4 \kappa}{\pi}\right)}\left[\kappa(1-\cos (\tilde{\omega} t))+\frac{\sin (\tilde{K} \tilde{\omega} t)-\tilde{K} \sin (\tilde{\omega} t)}{1-\tilde{K}^{2}}\right], \quad 0 \leq t \leq L / V,
$$

and Eq. (30) is only valid providing that the speed is not the critical one, i.e. $\tilde{K} \neq 1$.

As explained before, the amplitude of the free oscillations is determined by the sum of the amplitudes and the slopes of Equation (30) at $t=L / V$. Therefore, the only possibility for the free vibrations to vanish completely is that the amplitude and slope of the homogeneous solution is opposite to the amplitude and slope of the particular solution at $t=L / V$. This implies the two following conditions:

$$
\begin{aligned}
& \xi(t=L / V)=0 \Leftrightarrow \sin \left(\frac{\pi}{2 \tilde{K}}\right)\left(\kappa \sin \left(\frac{\pi}{2 \tilde{K}}\right)-\frac{\tilde{K}}{1-\tilde{K}^{2}} \cos \left(\frac{\pi}{2 \tilde{K}}\right)\right)=0, \\
& \dot{\xi}(t=L / V)=0 \Leftrightarrow \cos \left(\frac{\pi}{2 \tilde{K}}\right)\left(\kappa \sin \left(\frac{\pi}{2 \tilde{K}}\right)-\frac{\tilde{K}}{1-\tilde{K}^{2}} \cos \left(\frac{\pi}{2 \tilde{K}}\right)\right)=0 .
\end{aligned}
$$

Since equations (31) and (32) must be satisfied simultaneously, they can be reduced to the following one by intersecting its solutions: 
$\kappa \sin \left(\frac{\pi}{2 \tilde{K}}\right)-\frac{\tilde{K}}{1-\tilde{K}^{2}} \cos \left(\frac{\pi}{2 \tilde{K}}\right)=0$.

From Eq. (33) the cancellation values $\tilde{K}_{i}^{c}$ are obtained, while the corresponding real speeds $\tilde{V}_{i}^{c}$ are computed with Eq. (29). This approximate condition of cancellation was obtained in [19] considering an elastically supported beam subjected to a series of equidistant loads, but Eq. (33) shows that cancellation takes place for every single load at certain speeds, and therefore here it is shown to be independent of the number or spacing of the loads.

It is of interest to compare the true speeds for cancellation obtained for S-S and E-S beams, particularly for the fundamental mode $\left(V_{1 i}^{c}\right)$. From Eqs. (4) and (19) one gets, for $n=1$

$\frac{\bar{V}_{1 i}^{c}(\kappa)}{V_{1 i}^{c}}=\frac{\lambda_{1}(\kappa)}{\pi} \frac{\bar{K}_{1 i}^{c}(\kappa)}{K_{1 i}^{c}}$,

which is a relation between the exact results. Now taking the approximate solutions $\tilde{K}_{1 i}^{c}$ of Eq. (33), and recalling Eqs. (4) and (29)

$\frac{\tilde{V}_{i}^{c}}{V_{1 i}^{c}}=\frac{\tilde{K}_{i}^{c}}{K_{1 i}^{c}} \sqrt{\frac{\pi+4 \kappa}{\pi+8 \kappa+2 \pi \kappa^{2}}}$.

The results from Eqs. (34) and (35) are depicted in Fig. 8 for $\kappa$ varying between 0 and 0.3, for illustration purposes. It can be concluded that the approximate speeds of cancellation for E-S beams are in good agreement with the exact ones. Also, for the first mode the variation of the cancellation speeds observed with respect to the $\mathrm{S}-\mathrm{S}$ case is small for the low values of $\kappa$ usual in short to medium-span bridges.

\section{TRANSVERSE VIBRATION OF BEAMS UNDER A TRAIN OF MOVING LOADS: CANCELLATION OF RESONANCE}

When a beam is subject to the passage of a train of loads, and the spacing between these loads (or between groups of them) is repetitive, the free vibrations generated by each load can accumulate and create a resonance situation. If the beam undergoes one, two, three, etc. cycles of oscillation between the pass of consecutive groups of loads, then the phenomenon is called first resonance, second resonance and so on. The speeds of the consecutive resonances are thus submultiples of the first one or first resonant speed.

Conversely, the phenomenon of cancellation is independent of the spacing between the axle loads, and it takes place at fixed velocities for a given beam. Therefore, it may happen that the resonance and cancellation velocities coincide, a fact which can even be induced (at least theoretically) by adjusting the 
distance between the repetitive loads. If this is so, each load produces no free vibrations and these cannot accumulate. If the resonance speed does not coincide exactly with cancellation, but it is in the neighbourhood of cancellation, very little free vibrations will be produced and one should not expect a very strong resonance.

In the following sections the necessary conditions for resonance and cancellation to coincide are analysed, both for S-S as well as for E-S beams. This coincidence will be named hereafter as cancellation of resonance.

\subsection{Simply-supported beams}

Let $d$ be the characteristic distance between repeated loads of a train (see Fig. 9). The associated resonance speeds corresponding to the bending modes are the ones which lead to accumulation of free vibrations. It is known that these speeds can be easily calculated with the following formula:

$V_{n j}^{r}=\frac{d}{j \cdot T_{n}}=\frac{\omega_{n} d}{2 \pi j}, \quad n, j \geq 1$

Thus, the corresponding nondimensional speeds are

$K_{n j}^{r}=\frac{n \pi V_{n j}^{r}}{\omega_{n} L}=\frac{n d}{2 j L}, \quad n, j \geq 1$.

If the speeds given by Eq. (37) coincide with any of the cancellations predicted by Eq. (11), then resonance will be totally suppressed. Equating both expressions one obtains

$$
K_{n j}^{r}=K_{n i}^{c} \Rightarrow \frac{n d}{2 j L}=K_{n i}^{c} \Rightarrow\left(\frac{L}{d}\right)_{n j i}^{c}=\frac{n}{2 j K_{n i}^{c}}, \quad n, j, i \geq 1,
$$

and, recalling Eq. (11):

$\left(\frac{L}{d}\right)_{n j i}^{c}=\frac{n \pm 2 i}{2 j}>0, \quad n, j, i \geq 1$

Eq. (39) is a new result which gives the value of the $L / d$ ratio which causes the $i$ th cancellation of the $j$ th resonance for the $n$th mode. If the minus sign in the numerator is used, and still a positive $L / d$ ratio is obtained, index $i$ is said to take a "negative" value as in section 2.1.2. Values of the $L / d$ ratio close to the ones given by Eq. (39) will not cause a total cancellation of resonance, but the expected dynamic amplification will be low.

The most interesting cancellations of resonance are the ones associated to the first bending mode; these are obtained by substituting $n=1$ and retaining always the plus sign in Eq. (39). Their values are gathered in 
Table 3.

\subsection{Beams on elastic supports}

The resonant condition in terms of the velocity of a train of loads in an E-S beam is equal to that of a S-S beam, Eq. (36), except for the beam frequency $\bar{\omega}_{n}$ which depends on the supports stiffness. The corresponding nondimensional speeds are

$$
\begin{aligned}
\bar{K}_{n j}^{r}=\frac{\lambda_{n} \bar{V}_{n j}^{r}}{\bar{\omega}_{n} L}=\frac{\lambda_{n}}{2 \pi j} \frac{d}{L} & n, j \geq 1 \\
K_{n j}^{r}=\frac{\bar{K}_{n j}^{r} \lambda_{n}}{n \pi}=\left(\frac{\lambda_{n}}{n \pi}\right)^{2} \frac{n d}{2 j L} & n, j \geq 1
\end{aligned}
$$

When the speeds provided by Eq. (40a) coincide with any of the roots of Eq. (24) the resonance is cancelled, i.e

$$
\bar{K}_{n j}^{r}=\bar{K}_{n i}^{c} \Rightarrow\left(\frac{L}{d}\right)_{n j i}^{c}=\frac{\lambda_{n}}{2 \pi j} \frac{1}{\bar{K}_{n i}^{c}}=\left(\frac{\lambda_{n}}{n \pi}\right)^{2} \frac{n}{2 j K_{n i}^{c}} \quad n, j, i \geq 1
$$

As in Eq. (38), Eq. (41) provides the ratios $L / d$ causing the $i$ th cancellation of the $j$ th resonance of the $n$th mode for a E-S supported beam. In Table 3 these ratios are provided for the first four cancellations of the first four resonances considering $n=1, \kappa=0.1,0.2$. Notice that the values $K_{1 i}^{c}$ do not exhibit strong variations with $\kappa$ (see Table 1), but the effects of the elastic supports in Eq. (41) is amplified due to the presence of factor $\left(\lambda_{1} / \pi\right)^{2}$.

\section{TRANSVERSE VIBRATION OF BEAMS UNDER A TRAIN OF MOVING LOADS: MAXIMUM RESONANCE}

The situation opposite to cancellation of resonance takes place when the resonant speed coincides with one of the velocities of maximum free vibration amplitude. In such case vibrations of the largest possible amplitude will accumulate cycle after cycle, giving rise to the worst possible scenario, or maximum resonance. Because no closed-form expression of the speeds of maximum free vibration can be obtained for S-S beams, this section analyses jointly the conditions for maximum resonance to occur in both S-S as well as E-S beams. The discussion is limited to the fundamental bending mode.

The maximum resonance can take place in two circumstances. First, when the speed parameter for resonance, given by Eqs. (40) with $n=1$, coincides with any of the roots $\bar{K}_{1 i}^{m}$ of Eq. (23). Notice that in section 2.1.1 it was shown (for $\kappa=0$ ) that the root $\bar{K}_{11}^{m}$ is not attainable for nowadays railways; for small values of $\kappa$ one can expect the same conclusion to hold. In the second place, a strong resonance can also 
occur if the nondimensional speed is located in the ascending part of the free vibration plots, because the amplitude in this region can be very large; see for instance the region $K>\hat{K}_{1}^{m}$ in Fig. 2.

The first condition may be expressed as

$$
\begin{array}{lll}
K_{1 j}^{r}=\frac{\bar{K}_{1 j}^{r} \lambda_{1}}{\pi}=\left(\frac{\lambda_{1}}{\pi}\right)^{2} \frac{d}{2 j L}=K_{1 i}^{m} & j \geq 1 & i \geq 2, \\
\left(\frac{L}{d}\right)_{1 j i}^{m}=\left(\frac{\lambda_{1}}{\pi}\right)^{2} \frac{1}{2 j K_{1 i}^{m}} & j \geq 1 & i \geq 2 .
\end{array}
$$

Table 4 gathers some original results from this investigation: $L / d$ ratios for maximum resonance, obtained from Eq. (42b), are included for $\kappa=0.1,0.2$. The smaller the value of $i$, the larger the resonance amplitude. Because in real bridges damping is always present and it causes free vibrations to decay between each cycle of passage of loads, it can also be affirmed that in Table 4, the smaller the value of $j$ the larger the resonance amplitude. Eq. (42b) furnishes the locally maximum resonances, in the sense that $K_{1 i}^{m}$ are local maxima of the free vibration.

Additionally, the second situation is to be expected for values of $K_{1 j}^{r}$ higher than $\hat{K}_{1}^{m}(\kappa)$ (see Table 1). This can be referred to as an overall maximum resonance, because it is formed by addition of free vibrations of the largest possible amplitude. The associated upper limits of $L / d$ ratio can be computed as follows:

$K_{1 j}^{r}=\left(\frac{\lambda_{1}}{\pi}\right)^{2} \frac{d}{2 j L}>\hat{K}_{1}^{m} \quad j \geq 1$,

$\left(\frac{L}{d}\right)_{1 j}^{m}<\left(\frac{\lambda_{1}}{\pi}\right)^{2} \frac{1}{2 j \hat{K}_{1}^{m}} \quad j \geq 1$

The upper limits for the overall maximum resonance to happen, Eq. (43b), are also shown in Table 4 for the first time. If the speed of the railway line is lower than the corresponding resonance speeds, Eq. (36), maximum resonances of course cannot take place.

\section{CASE STUDY}

For illustration purposes, a simply supported bridge of span $L=27 \mathrm{~m}$, linear mass $m=15000 \mathrm{~kg} / \mathrm{m}$ and fundamental frequency $\omega_{1}=43.98 \mathrm{rad} / \mathrm{s}(7 \mathrm{~Hz})$ is analysed. These are realistic values for a single-track, composite high-speed bridge. According to Eurocode 1, for such a structure damping should be taken equal to $\zeta=0.5 \%$ in a dynamic analysis. The bridge is subject to the passage of train A1, which is one of the HSLM-A Universal Trains also defined in Eurocode 1, with a characteristic distance $d=18 \mathrm{~m}$ (Fig. 9). Therefore, $L / d=1.5$. According to Table 3 , such value of the $L / d$ ratio produces cancellation of the first 
resonance of the fundamental mode, as it is well known (see for instance references [4] and [16]). Nevertheless, this value is very close to one of the ratios which create a maximum resonance, as it can be seen in Table 4 for $\kappa=0: L / d=1.482$ corresponds to a second resonance $(j=2)$ of the fundamental mode created by addition of free vibrations generated in the third maximum of free vibrations $(i=3)$. The maximum acceleration along the beam is plotted in Fig 10 for speeds ranging from 100 to $500 \mathrm{~km} / \mathrm{h}$ in steps of $1.8 \mathrm{~km} / \mathrm{h}$ : the first five bending modes have been taken into account, and the maximum acceleration is computed among 19 equally spaced sections: $x / L=\{0.05,0.10,0.15,0.20, \ldots 0.90,0.95\}$.

As it was expected, no significant amplification is generated at the first resonance speed $V_{11}^{r}=(43.98 \cdot 18 / 2 \pi) \cdot 3.6=453.6 \mathrm{~km} / \mathrm{h}$ but, conversely, a marked resonance is seen at $V_{12}^{r}=V_{11}^{r} / 2=226.8 \mathrm{~km} / \mathrm{h}$ which produces a maximum vertical acceleration of $4.9 \mathrm{~m} / \mathrm{s}^{2}$, precisely at the mid-span section $x / L=0.5$.

In the next section a formula is proposed for estimating the amplitude of the resonant acceleration peaks as the one shown in Fig. 10.

\section{APPROXIMATE FORMULA FOR THE VERTICAL ACCELERATION}

The maximum vertical acceleration has become a most important parameter to keep under control in highspeed railway bridges, especially in medium or short S-S spans. It is therefore of great interest to obtain a simple formula to estimate the value of this parameter during the initial design stages.

For S-S bridges, the ideas exposed in this paper can be combined in a simple manner to produce such a formula. To this end, the following assumptions are made: (i) the contributions of modes higher than the fundamental are neglected; (ii) damping ratio $\zeta$ is small enough as to accept $\zeta \ll<1$ and $\zeta^{2} \ll \zeta$. The objective is to add the contributions of the successive passing loads at resonance, each of which produces free vibrations of amplitude given by an expression similar to Eq. (7).

\subsection{Amplitude of the free vibrations for light damping}

For the first mode, structural damping slightly modifies the differential equations that govern each modal amplitude, so that one gets the following well-know expressions:

$$
\begin{aligned}
& \ddot{\xi}(t)+2 \zeta \omega \dot{\xi}(t)+\omega^{2} \xi(t)=-\frac{2 P}{m L} \sin \frac{\pi V}{L} t, \quad 0 \leq t \leq L / V, \\
& \ddot{\xi}(t)+2 \zeta \omega \dot{\xi}(t)+\omega^{2} \xi(t)=0, \quad t>L / V .
\end{aligned}
$$

The solution to Eq. (44b) is a damped sinusoid of initial amplitude $\delta$. As in section 2.1, this initial amplitude can be easily computed from the solution to Eq. (44a) and its derivative at time $t=L / V$. For small damping (tipically $\zeta<0.03$ ) one gets another well-known result

$$
\delta=\frac{2 P}{m L \omega^{2}} R(K, \zeta)
$$


where

$$
R(K, \zeta)=\frac{K}{1-K^{2}} \sqrt{1+\mathrm{e}^{-\frac{\zeta \pi}{K}}\left(\mathrm{e}^{-\frac{\zeta \pi}{K}}+2 \cos \frac{\pi}{K}\right)} .
$$

Consequently, in the presence of low damping the initial amplitude of the acceleration at mid-span, for $t>$ $L / V$, can be approximated simply by multiplying twice by the natural frequency:

$a \square \omega^{2} \delta=\frac{2 P}{m L} R(K, \zeta)$.

\subsection{Combined effects of closely spaced loads}

In conventional railways (not Maglevs), the total weight of the train is distributed in groups of closely spaced loads corresponding to the so-called trucks or bogies. For the sake of dynamic calculations, in high-speed bridges it is customary to use articulated trains, which share a bogie located between each pair of coaches. Therefore, the repetitive loads which create resonance are arranged in pairs (see Fig. 9). Probably, the most popular articulated train prescribed by regulations is the HSLM-A from Eurocode [13]; this load model comprises ten different trains A1, A2 ... A10 of varying axle load, car length, etc., very similar to the one shown in Fig. 9.

In general, if the loads are grouped in closely spaced ensembles, there will be a small decay of structural vibration between consecutive loads of the ensemble. Neglecting this decay-which is due to the presence of damping - is a slightly conservative approximation. Therefore, one can compute the combined free vibrations generated by such groups of loads by directly adding their out-of-phase amplitudes as follows. It is known that

$$
\sum_{k=0}^{N} \cos (\varphi+k \alpha)=\frac{\sin \left(\frac{(N+1) \alpha}{2}\right)}{\sin \left(\frac{\alpha}{2}\right)} \cos \left(\varphi+\frac{N \alpha}{2}\right)
$$

therefore, assuming that each load generates free vibrations of amplitude " $a$ " and the number of loads of the group is $N_{b}$ (typically $N_{b}=2$ ), then the total free vibration can be approximated as

$$
\sum_{k=0}^{N_{b}-1} a \cdot \cos \left(\omega\left(t-\frac{k b}{V}\right)\right)=-a \cdot \frac{\sin \frac{\omega b N_{b}}{2 V}}{\sin \frac{\omega b}{2 V}} \cdot \cos \left(\omega t+\left(1-N_{b}\right) \frac{\omega b}{2 V}\right),
$$

where $b$ is the (short) distance between consecutive loads. This is equivalent to saying that the amplitude $\hat{a}$ of the combined free vibration caused by a group of $N_{b}$ loads, regardless of its sign, is approximately equal to 
$\hat{a}=a \cdot\left|\frac{\sin \frac{\omega b N_{b}}{2 V}}{\sin \frac{\omega b}{2 V}}\right|$.

\subsection{Addition of damped free vibrations at resonance}

Finally, if one train consists of $N$ groups of $N_{b}$ loads, one has to apply superposition in order to add the damped free vibrations generated by all of them. The worst possible scenario is resonance, which implies that the vibrations are out of phase by an exact integer multiple $j$ of the fundamental period $T=2 \pi / \omega$. If the characteristic distance between the groups of loads is $d$, the resonant speed for the first mode is

$V_{1 j}^{r}=\frac{d}{j \cdot T}=\frac{\omega d}{2 \pi j}, \quad j=1,2,3, \ldots$,

and the nondimensional speed is

$K_{1 j}^{r}=\frac{d}{2 j L}, \quad j=1,2,3, \ldots$

Accordingly, for the $j$ th resonance speed, Eq. (50) can be rewritten as

$\hat{a}=a \cdot F_{B}\left(d, N_{b}, b, j\right) \quad F_{B}\left(d, N_{b}, b, j\right)=\left|\frac{\sin \frac{j \pi b N_{b}}{d}}{\sin \frac{j \pi b}{d}}\right|$.

We shall refer to $F_{B}$ as bogie factor in what follows. If $N_{b}=2, F_{B}(d, 2, b, j)=2 \cos (j \pi b / d)$. For convenience time can be set equal to zero at the precise instant when the first group of loads leaves the beam and the acceleration (free vibration) generated by these loads reaches its first maximum amplitude. Therefore, an appropriate cosine function is used in Eq. (54). This first maximum amplitude will be slightly smaller than $\hat{a}$ because of damping, but the difference will be small because no more than one half-cycle will have passed since the last load of the group has left the beam. Since the speed is given by Eq. (51), the subsequent $N^{-1}$ groups of loads cause a vibration which adds to the first one as follows:

$\sum_{k=0}^{N-1} \hat{a} \cdot \mathrm{e}^{-\zeta \omega\left(t-k \frac{d}{V_{1 j}^{r}}\right)} \cos \left(\omega\left(t-k \frac{d}{V_{1 j}^{r}}\right)\right)=\sum_{k=0}^{N-1} \hat{a} \cdot \mathrm{e}^{-\zeta \omega(t-k j T)} \cos (\omega(t-k j T))$,

The maximum acceleration due to the combination of the free vibrations will take place when the last group of loads has left the beam and the acceleration created by this group reaches its maximum. In this precise moment, $t=j(N-1) T$ and the sum is 
$\hat{a} \sum_{k=0}^{N-1} \mathrm{e}^{-\zeta \omega(j(N-1) T-k j T)} \cos (\omega(j(N-1) T-k j T))=\hat{a} \sum_{k=0}^{N-1} \mathrm{e}^{-\zeta 2 \pi j(N-1-k)}=\hat{a} \frac{\mathrm{e}^{2 \pi \zeta j}-\mathrm{e}^{-2 \pi \zeta j(N-1)}}{\mathrm{e}^{2 \pi \zeta j}-1}$.

The last factor in Eq. (55) will be referred to as superposition factor as it represents the addition of the effects of $N$ loads at the $j$ th resonance:

$F_{S}(\zeta, N, j)=\frac{\mathrm{e}^{2 \pi \zeta j}-\mathrm{e}^{-2 \pi \zeta j(N-1)}}{\mathrm{e}^{2 \pi \zeta j}-1}$

\subsection{Approximate formula for the vertical acceleration}

Combining Eqs. (46), (47), (53) and (55), a new formula can be derived for estimating the acceleration due to resonances of the fundamental mode. This is obtained as the product of the free vibration amplitude times the bogie factor and times the superposition factor:

$$
\begin{aligned}
& a_{\text {res }}=\frac{2 P}{m L} R(K, \zeta) \cdot F_{B}\left(d, N_{b}, b, j\right) \cdot F_{S}(\zeta, N, j) \quad K=K_{1 j}^{r}=\frac{d}{2 j L} \\
& a_{\text {res }}=\frac{2 P}{m L} \frac{K}{1-K^{2}} \sqrt{1+\mathrm{e}^{-\frac{\zeta \pi}{K}}\left(\mathrm{e}^{-\frac{\zeta \pi}{K}}+2 \cos \frac{\pi}{K}\right)} \cdot\left|\frac{\sin \frac{j \pi b N_{b}}{d}}{\sin \frac{j \pi b}{d}}\right| \cdot \frac{\mathrm{e}^{2 \pi \zeta j}-\mathrm{e}^{-2 \pi \zeta j(N-1)}}{\mathrm{e}^{2 \pi \zeta j}-1} \quad K=K_{1 j}^{r}=\frac{d}{2 j L}
\end{aligned}
$$

There may be situations when the true maximum acceleration is obtained during the forced vibration period, i.e. while the last group of loads, or some of them are still crossing the bridge. Moreover, the characteristic distance $d$ usually varies between coaches and locomotives, and therefore the resonance phenomenon is altered to some extent. However, the largest part of the resonant response will be created by the repetitive passenger coaches, and this effect will be captured by Eqs. (57). Especially due to the presence of the rear locomotives, it is difficult to predict with simple formulas whether the acceleration will be somewhat increased or decreased. Either one thing or the opposite may happen. Nevertheless, Eqs. (57) are still useful because they are simple enough to be used in a spreadsheet or a calculator, and one can always take conservative values of $N$, even artificially increased in one or two units, simulating that the train is composed by a larger number of coaches.

The approach adopted for obtaining Eqs. (57) is similar to the LIR method developed by the SNCF within the ERRI D-214 Committee [18], although there are two main differences which consequently lead to different formulas. The first and most important difference is that the LIR method computes and accumulates the amplitude of the free vibrations due to each load at any circulation speed, i.e. it is not restricted to resonance speeds as Eqs. (57) are. Therefore, the LIR method is more versatile, but simultaneously its mathematical expression is somewhat more involved: in the LIR method one finds summations of damped sine and cosine functions, while the equivalent in Eqs. (57) is the superposition factor. Secondly, in Eqs. (57) the effect of each group of closely spaced loads (namely, the bogies) is 
computed by combining the effects of all the loads in the group and not taking account of damping; as section 6.2 describes, this slightly conservative approach gives rise to the bogie factor in Eqs. (57). In summary, the approximate equations presented here are based in the same principle as the LIR method (addition of free vibrations) but their objective is to obtain directly the maximum peak values, which correspond to the resonant speeds of the first mode $(K=d /(2 j L))$ where all the free vibrations accumulate in phase.

In this regard, one should take into account the case when a train produces resonance at speed $V_{1 j}^{r}$ only slightly greater than the design speed of the line $\left(V_{\max }\right)$. In such cases the maximum resonant response will not be attained, but a strong response should be expected precisely at $V_{\max }$. For this reason, one should artificially increase $V_{\max }>V_{1 j}^{r}$, apply Eqs. (57) for $V_{1 j}^{r}$, and obtain the resulting value of $a_{r e s}$ in order to assess the importance of such resonance.

\subsection{Practical application and validation}

As a particularly useful application, one can apply Eqs. (57) to obtain an estimate of the acceleration due to the free vibrations generated by high-speed load model HSLM-A. The exact results can be obtained by closed-form integration of Eqs. (44). This solution is available from many authors as, for instance, [1, 4, 16]. For a $20 \mathrm{~m}$ bridge, Fig. 11 shows the comparison between the maximum acceleration along the beam (obtained with the first five bending modes) and the one approximated with Eqs. (57). As in section 5, the maximum acceleration is computed among 19 equally spaced sections: $x / L=\{0.05,0.10,0.15,0.20, \ldots$ $0.90,0.95\}$. The linear mass of the bridge is $20000 \mathrm{~kg} / \mathrm{m}$, its fundamental frequency $7.04 \mathrm{~Hz}$ and damping $\zeta=0.01$. The number of groups of loads $N$ is always taken equal to the number of cars (coaches+ locomotives) plus one (last bogie). As it can be seen, the predominant peaks of resonant behaviour are very well approximated. In this example most of them correspond to second and third resonances $(j=2,3)$ of the fundamental mode. The first and second cancellations of the first mode can be also detected at $V=$ $3.6 \cdot(1 / 3) \cdot 2 \cdot 20 \cdot 7.04 \approx 338 \mathrm{~km} / \mathrm{h}$ and $V=3.6 \cdot(1 / 5) \cdot 2 \cdot 20 \cdot 7.04 \approx 203 \mathrm{~km} / \mathrm{h}$. The resonant peak from the previous case study, Fig. 10, is also well approximated by Eqs. (57), which produce a value of $5.1 \mathrm{~m} / \mathrm{s}^{2}$.

However, in Fig. 11 it is seen that the maximum response created by train A8 at $316.8 \mathrm{~km} / \mathrm{h}(88 \mathrm{~m} / \mathrm{s})$ is underestimated by $30 \%$ approximately $\left(2.3 \mathrm{~m} / \mathrm{s}^{2}\right.$ instead of $\left.3.3 \mathrm{~m} / \mathrm{s}^{2}\right)$. This peak is generated at $x=0.65 \cdot L$ by the addition of a second resonance of the fundamental mode $\left(2.2 \mathrm{~m} / \mathrm{s}^{2}\right)$ plus a contribution of the second mode of $0.9 \mathrm{~m} / \mathrm{s}^{2}$, and smaller contributions of higher modes. The relative importance of the second mode in this case is explained because the (homogeneous) free vibrations generated by the twinaxle bogies which leave the bridge combine, at certain speeds, with the homogeneous part of the forced vibrations generated by the two loads of the following bogie when they enter the bridge. The speeds when this kind of combination will produce maximum acceleration can not be predicted by formulas as simple as Eq. (36) because the homogeneous forced and free accelerations can not be superimposed straightforwardly: the last maximum of homogeneous forced acceleration need not necessarily be 
separated one exact period from the first maximum of homogenous free acceleration (this is evident because the acceleration created by a single load is not differentiable at $t=L / V$ ). For this particular $20 \mathrm{~m}$ bridge one can calculate the response to train A8 due exclusively to the second bending mode and a sharp peak is detected precisely at $88 \mathrm{~m} / \mathrm{s}$, which is the $2^{\text {nd }}$ order resonance of the first mode for $d=25 \mathrm{~m}$ and thus it is also the $8^{\text {th }}$ order resonance for the second mode. Although they are not perfectly in phase, the resulting effect is that the two axles leaving the bridge combine with the vibrations generated by the next two axles entering the bridge, and thus the vibration increases during 4 cycles of the second mode; then it decreases during 4 more cycles and the remaining residual vibration adds to the next 4 cycles of increasing vibration.

These particular kinds of resonance phenomena are known to the bridge engineers, and they may have an influence in certain cases as Fig. 11 shows for the A8 train. This is one of the reasons why it is necessary to carry out complete vibration analyses in real bridges designed for high-speed traffic. Nevertheless, Eqs. (57) do not intend to substitute such detailed analyses, but to give an estimate of the maximum resonant acceleration based in the fundamental mode contribution. In order to prove that this estimate can be useful for practical purposes, a comprehensive validation has been carried out by analysing 33 bridges shown in Fig. 3. For each of the 11 different spans $(10,12,14,16, \ldots 30$ meters $)$, three frequencies have been selected: they correspond to the upper and lower limits of the band, and a third value which is equal to the average. This range of span lengths is a usual one for S-S high speed bridges; Eqs. (57) are not limited to this range, but longer bridges are not so easily affected by excessive vertical accelerations, and shorter ones are often built as rigid frames rather than simply supported. The linear mass $(\mathrm{kg} / \mathrm{m})$ assigned to each bridge is equal to its span (m) multiplied by 1000 . The 33 bridges have been analysed under the passage of the HSLM-A for speeds from $100 \mathrm{~km} / \mathrm{h}$ up to $350 \cdot 1.2=420 \mathrm{~km} / \mathrm{h}$, according to Eurocode [13] (specifically, speeds from 28 to $117 \mathrm{~m} / \mathrm{s}$ in steps of $1 \mathrm{~m} / \mathrm{s}$ have been used). Purposely, load model HSLMA has been designed with characteristic distances $d$ (coach length) ranging from 18 to $27 \mathrm{~m}$; as it was mentioned in section 2.1.2, it is therefore expected that some HSLM-A train will create a predominant effect of resonance of the fundamental mode.

Two different types of analyses have been performed: first, only the fundamental mode has been considered; secondly, the first five modes have been used for computing the maximum acceleration along the beam as in Fig. 11. Three different damping ratios have been considered for each bridge: 0.01, 0.015 and 0.02 , and the maximum peak acceleration at resonance due to the most unfavourable HSLM-A train has been computed. This is the determinant value for the verification of the Serviceability Limit State ( $a_{\max }$ $<3.5 \mathrm{~m} / \mathrm{s}^{2}$ for ballasted tracks). Table 5 contains a summary of the main results: for each bridge, the values shown on the table correspond to the damping ratio which gives a higher relative error in the analysis performed with five modes. Therefore, for certain combinations of span and damping the analysis with one mode produces an error larger than the one shown on the table, but never greater than $-10 \%$ / $+12 \%$, which can be considered a very good first approximation and proves the utility of these formulas 
for practical purposes. When the first five modes are taken into account, the error usually (but not always) increases and its bounds are $-12 \% /+9 \%$ except for one of the $18 \mathrm{~m}$ bridges, where a local increase of the vibrations induced by the last locomotive takes place simultaneously with a maximum of a cycle of oscillation of the third mode (of frequency equal to $69.1 \mathrm{~Hz}$ ). This particular coincidence causes the last resonance cycle to have a slightly larger amplitude than predicted by Eqs. (57), which in this case underestimate the maximum peak response in $18.4 \%$. The approximated values from Eqs. (57), however, can still be considered useful and satisfactory for a first assessment.

On Table 5 it is seen that the peak acceleration of some bridges corresponds to a given train when the analysis is performed with one mode, but to a different one when five modes are taken into account (e.g. the $20 \mathrm{~m}$ bridge of the lowest frequency). This occurs only in some of the cases analysed, when the vertical accelerations created by similar trains are but slightly different. In such cases, the contributions of higher frequency modes may cause the predominant train to be exceeded by one of its neighbours from the HSLM-A scheme. However, as it can be seen in the table, the differences in these cases are always small (even if one compares the acceleration computed with one or five modes). In this regard, it has been verified that Eqs. (57) predict correctly the train which creates the highest peak acceleration in the analyses carried out with one mode.

\section{CONCLUSIONS}

The free vibrations created on simply and elastically supported beams due to the pass of concentrated, constant-valued moving loads have been analysed in detail. From this analysis, the following conclusions can be drawn:

1) In absence of damping, the free vibrations created by a single moving load on simply supported beams are maximum for certain speeds, while they are null (cancellation) for other particular speed values. These facts were anticipated by Savin [4] and Pesterev et al [12]. The former showed that damping alters this fact but slightly. In the present paper new closed form expressions have been given for the cancellation speeds of a generic mode, as well as for the most representative points of maximum amplitude. Also, a new graphical interpretation of the cancellation phenomenon has been given in terms of the homogeneous and particular components of the forced vibration solution.

2) This research has shown that the preceding conclusions, as well as the interpretation of cancellation in terms of homogeneous and particular components, hold for elastically supported beams as well. As original results, values of nondimensional speed corresponding to maximum or zero amplitudes of free vibrations have been obtained for different supports stiffness. The variation observed with respect to the simply supported case is small for the first mode but increases for higher modes. 
3) An approximate expression of the cancellation speeds for an elastically supported beam, previously deduced by Yang et al. [19] from the vibrations created by a series of moving loads, has been derived in this paper on the basis of the homogeneous and particular components of the forced vibration caused by a single load. The cancellation speeds provided by this approximate formulation are in good agreement with the exact ones for the first mode.

4) Knowing a priori the speeds of maximum free vibrations or cancellation of each mode can be of great interest for experimental tests on road or railway bridges. Since in such tests it is very common to use a single vehicle (truck or locomotive) as excitation, it is desirable to obtain the highest possible vibration amplitudes in order to have good estimates of the modal properties of the bridge, particularly structural damping.

5) Series of equidistant loads or groups of loads crossing the bridge at certain speeds may induce resonance. If these resonance speeds coincide with either a maximum free vibration speed or a cancellation speed, then a maximum resonance or a cancellation of resonance will occur. This will happen for particular values of the $L / d$ ratio for each mode, which have been provided here comprehensively for the first time, both for simply as well as for elastically supported beams. For simply supported ones, the first resonance of the first mode is well-known to cancel when $L / d$ is equal or close to 1.5 , but here it has been shown that simultaneously $(L / d=1.482)$ the second resonance reaches a local maximum.

6) A new approximate formula for estimating the maximum acceleration caused by resonances of the fundamental mode has been proposed. The formula is based on the addition of free vibrations; it considers resonances of any order - any number of cycles between the pass of consecutive loadsand it takes also account of the out-of-phase contributions of different axles belonging to a same bogie. After extensive numerical testing, it has proved to be a useful tool for a first assessment of simply supported bridges according to building codes such as the Eurocode.

\section{ACKNOWLEDGEMENTS}

The authors acknowledge the financial support of the State Secretariat for Research of the Spanish Ministry of Science and Innovation (Secretaría de Estado de Investigación, Ministerio de Ciencia e Innovación, MICINN) in the framework of the Research Project BIA2008-04111. 


\section{REFERENCES}

[1] L. Frýba. Dynamics of solids and structures under moving loads. Noordhoff International Publishing, Groningen, The Netherlands, 1972 (3rd ed. by Thomas Telford, 1999).

[2] W. Weaver, S.P. Timoshenko, D.H. Young, Vibration problems in engineering (5th ed.). Wiley, New York. pp 448-454, 1990.

[3] G.T. Michaltsos, The influence of centripetal and Coriolis forces on the dynamic response of light bridges under moving vehicles. Journal of Sound and Vibration 247 (2), pp 261-277, 2001.

[4] E. Savin, Dynamic amplification factor and response spectrum for the evaluation of vibrations of beams under successive moving loads. Journal of Sound and Vibration 248(2), pp. 267-288, 2001.

[5] A. N. Kryloff, Über die erzwungenen Schwingungen von gleichförmigen elastischen Stäben (On the forced oscillations of uniform elastic rods). Mathematische Annalen. Mathematical Collection of Papers of the Academy of Sciences, (A. N. Kryloff, editor) Vol. 61. Peterburg: Matematischeskii sbornik Akademii Nauk, 1905.

[6] F. Bleich, Theorie und Berechnung der eisernen Brücken (Theory and analysis of iron bridges). Springer, 1924.

[7] S.P. Timoshenko, Vibration of bridges. Transactions Vol. 53, American Society of Mechanical Engineers, 1928.

[8] E.H. Lee, On a "paradox" in beam vibration theory. Quarterly Journal of Applied Mathematics 10, 290-292, 1952.

[9] L. Maunder, On the work of a force crossing a beam. Quarterly Journal of Applied Mathematics, 17(4), 437-440, 1960.

[10] M. Olsson, On the fundamental moving load problem. Journal of Sound and Vibration, 145(2), pp. 299-307, 1990.

[11] J.A. Gbadeyan, T. Oni, Dynamic behaviour of beams and rectangular plates under moving loads. Journal of Sound and Vibration 182(5), pp 677-695, 1995.

[12] A.V. Pesterev, B. Yang, L.A. Bergman, C.A. Tan, Revisiting the moving force problem. Journal of 
Sound and Vibration 261, pp. 75-91, 2003.

[13] EN 1991-2. European Committee for Standardisation (CEN), Eurocode 1: Actions on structures. Part 2: General actions - Traffic loads on bridges, 2003.

[14] Bolotin, V. V., The Dynamic Stability of Elastic Systems. Holden-Day, San Francisco, CA, 1964

[15] M. Klasztorny, J. Langer, Dynamic response of single-span beam bridges to a series of moving loads. Earthquake Engineering and Structural Dynamics 19, pp 1107-1124, 1990.

[16] Y.B. Yang, J.D. Yau, L.C. Hsu, Vibration of simple beams due to trains moving at high speeds. Engineering Structures, 19(11), pp. 936-944, 1997.

[17] L. Frýba, A rough assessment of bridges for high speed trains. Engineering Structures 23, pp 548$556,2001$.

[18] European Rail Research Institute, D-214 Committee (ERRI D-214), PontsRails pour vitesses > 200 km/h. Rapport final (ERRI D-214/RP9), 2000.

[19] Y.B. Yang, C.L. Lin, J.D.Yau, D.W. Chang, Mechanism of resonance and cancellation for train induced vibrations on bridges with elastic bearings, Journal of Sound and Vibration 269, 345-360, 2004.

[20] H. Xia, N. Zhang, W.W. Guo, Analysis of resonance mechanism and conditions of train-bridge system. Journal of Sound and Vibration 297, pp 810-822, 2006.

[21] Y.L. Xu, Q. Li, D.J. Wu, Z.W. Chen, Stress and acceleration analysis of coupled vehicle and longspan bridge systems using the mode superposition method. Engineering Structures, 32, pp 1356-1368, 2010.

[22] P. Museros, E. Alarcón, Influence of the second bending mode on the response of high-speed bridges at resonance. Journal of Structural Engineering 131, pp 404-415, 2005.

[23] J.D. Yau, Y.B. Yang, Vertical accelerations of simple beams due to successive loads travelling at resonant speeds. Journal of Sound and Vibration, 289, pp 210-228, 2006.

[24] L. Frýba. Dynamics of Railway Bridges. Thomas Telford. 1996

[25] P. Museros, E. Alarcón, An investigation on the importance of Train-Bridge interaction at resonance. 
6th International Conference on Computational Structures Technology, CIVIL-COMP, Prague, 2002.

[26] A. Doménech, P. Museros, Influence of the vehicle model on the response of high-speed railway bridges at resonance. Analysis of the additional damping method prescribed by Eurocode 1. 8th International Conference on Structures Dynamics, EURODYN, Leuven, 2011.

[27] D.J. Gorman, Free vibration analysis of beams and shafts, Wiley, 1975.

[28] I.A. Karnovsky, O.I. Lebed, Formulas for structural dynamics. Tables, graphs and solutions, McGraw Hill, 2000.

[29] A.S. Dmitriev, Transverse vibrations of a beam with elastic supports during motion of a concentrated force. International Applied Mechanics 10 (4), pp 113-118, 1974. 


\section{FIGURE CAPTIONS}

Fig. 1. Moving load problem. (a) Simply supported (S-S) and (b) elastically supported beam (E-S).

Fig. 2. Amplitude of the free vibrations (first mode).

Fig. 3. Usual range of spans and fundamental frequencies for railway bridges ( $\diamond$ bridges selected for analysis in section 6.5).

Fig. 4. Maximum expected values of the nondimensional speed $K$, corresponding to $V_{\max }=500 \mathrm{~km} / \mathrm{h}$.

Fig. 5. (a) General free vibration and (b) cancellation case. Homogeneous $(-)$, particular $(-)$, and total $(-)$ vibration.

Fig. 6. Possible cancellations for different bending modes of an S-S beam. Homogeneous ( -$)$, particular $(-)$, and total ( - ) vibration.

Fig. 7. (a) First and (b) second modal response amplitudes vs. speed parameter $K_{n}, n=1,2$ for values of $\kappa$ in the range $[0,0.2]$.

Fig. 8. Variation of the cancellation speeds with the supports flexibility. (a) First and second and (b) third and fourth cancellations of the fundamental mode.

Fig. 9. Train composed of equidistant groups of loads.

Fig. 10. Response of a $27 \mathrm{~m}$ bridge to A1 train from Eurocode $1(L / d=1.5)$.

Fig. 11. Response of a $20 \mathrm{~m} \mathrm{S-S} \mathrm{bridge} \mathrm{to} \mathrm{the} 10$ trains of HSLM-A load model ( resonance values approximated with Eqs. (57)). 


\section{TABLE CAPTIONS}

Table 1. Values of $K_{n i}^{m}$ and $K_{n i}^{c}$ corresponding to attainable maxima of the free vibrations and cancellations for the first two modes of an E-S beam.

Table 2. Values of $K_{n i}^{c}$ corresponding to cancellations of the first four modes ( $\mathrm{S}-\mathrm{S}$ beam).

Table 3. Values of $L / d$ corresponding to cancellations of the first four resonances of the first bending mode for $\kappa=\{0.00,0.10,0.20\}$.

Table 4. Values of $L / d$ corresponding to locally maximum resonances and upper limits associated to overall maximum resonances of the first bending for $\kappa=\{0.00,0.10,0.20\}$.

Table 5. Maximum vertical acceleration $a_{\max }\left(\mathrm{m} / \mathrm{s}^{2}\right)$ and comparison with $a_{\text {res }}$ obtained from Eqs. (57) for 33 test bridges. For each bridge $m(\mathrm{~kg} / \mathrm{m})=1000 \cdot L(\mathrm{~m})$. 

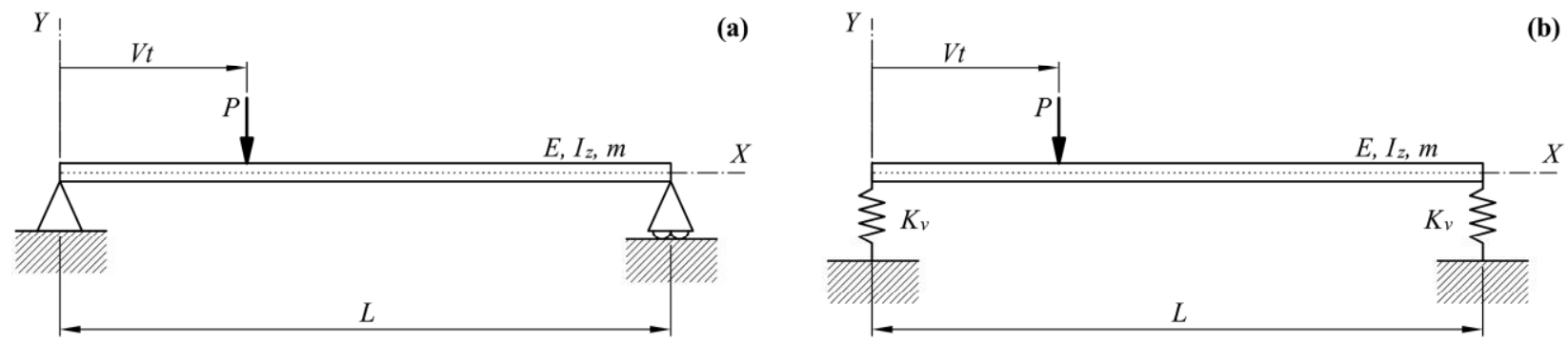

Fig. 1. Moving load problem. (a) Simply supported (S-S) and (b) elastically supported beam (E-S). 


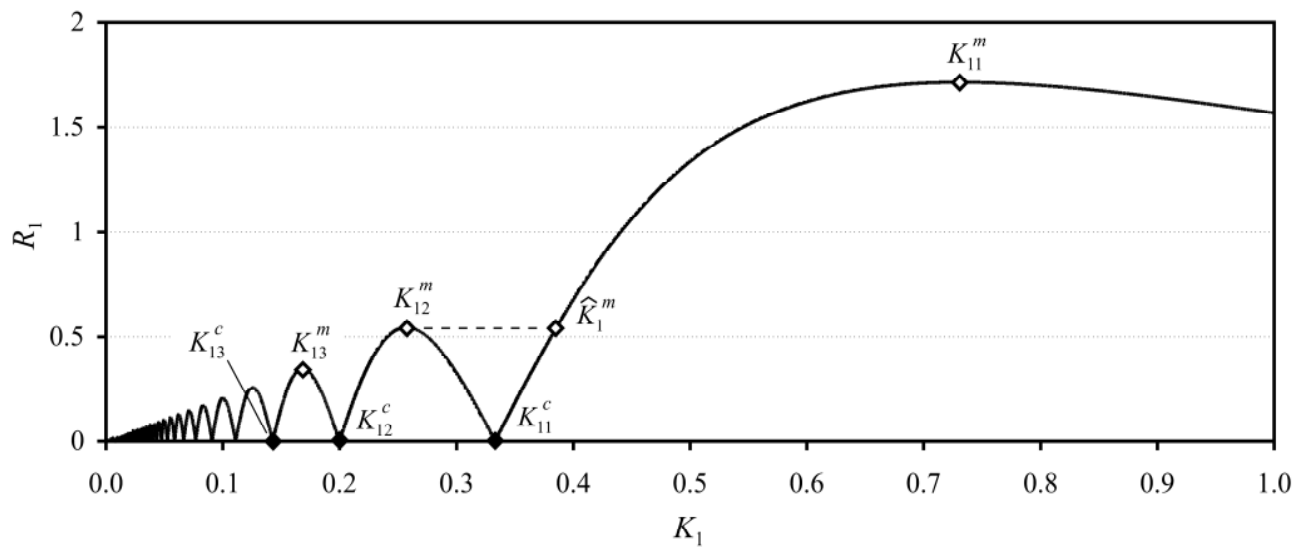

Fig. 2. Amplitude of the free vibrations (first mode). 


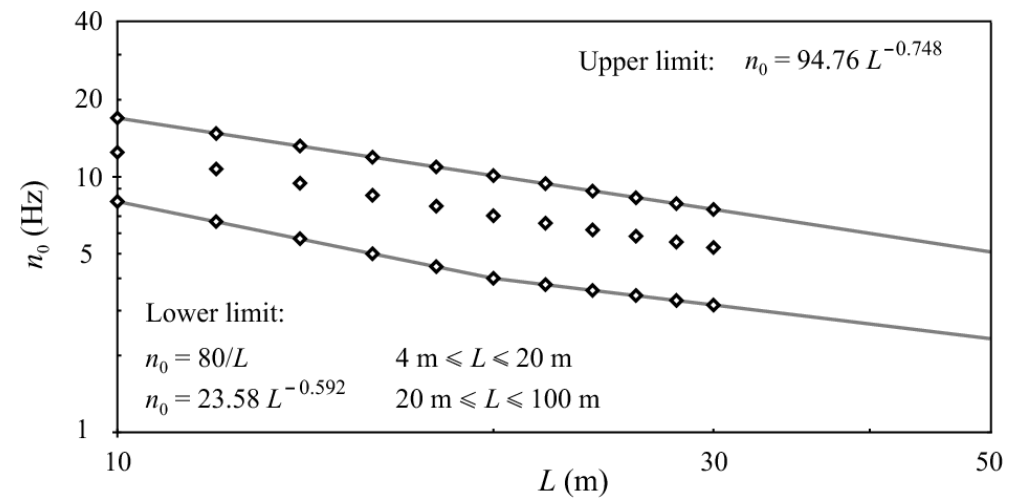

Fig. 3. Usual range of spans and fundamental frequencies for railway bridges ( $\diamond$ bridges selected for analysis in section 6.5). 


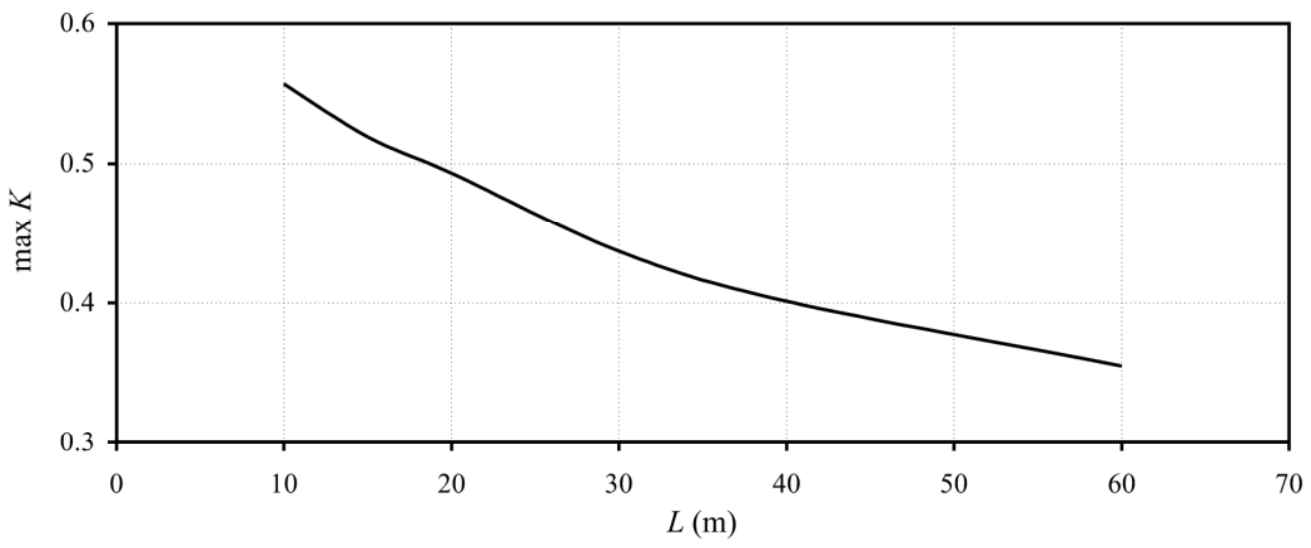

Fig. 4. Maximum expected values of the nondimensional speed $K$, corresponding to $V_{\max }=500 \mathrm{~km} / \mathrm{h}$. 


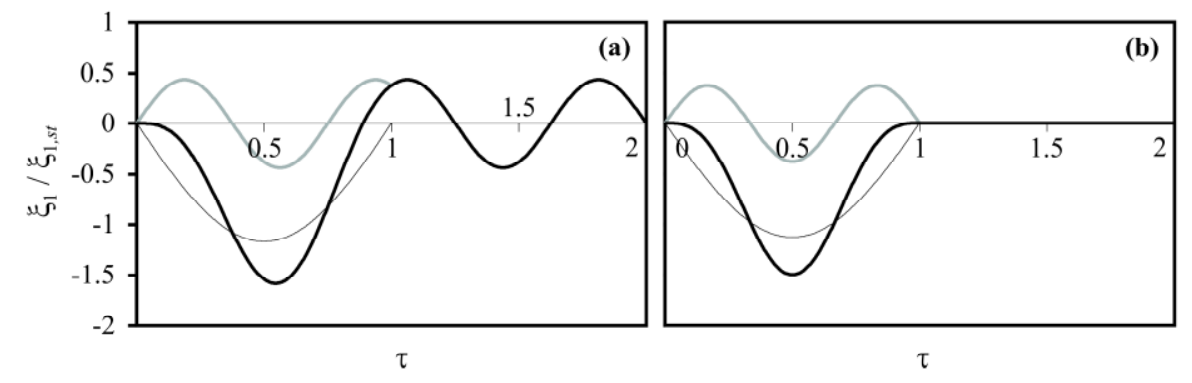

Fig. 5. (a) General free vibration and (b) cancellation case. Homogeneous ( - ), particular $(-)$, and total ( 一 ) vibration. 

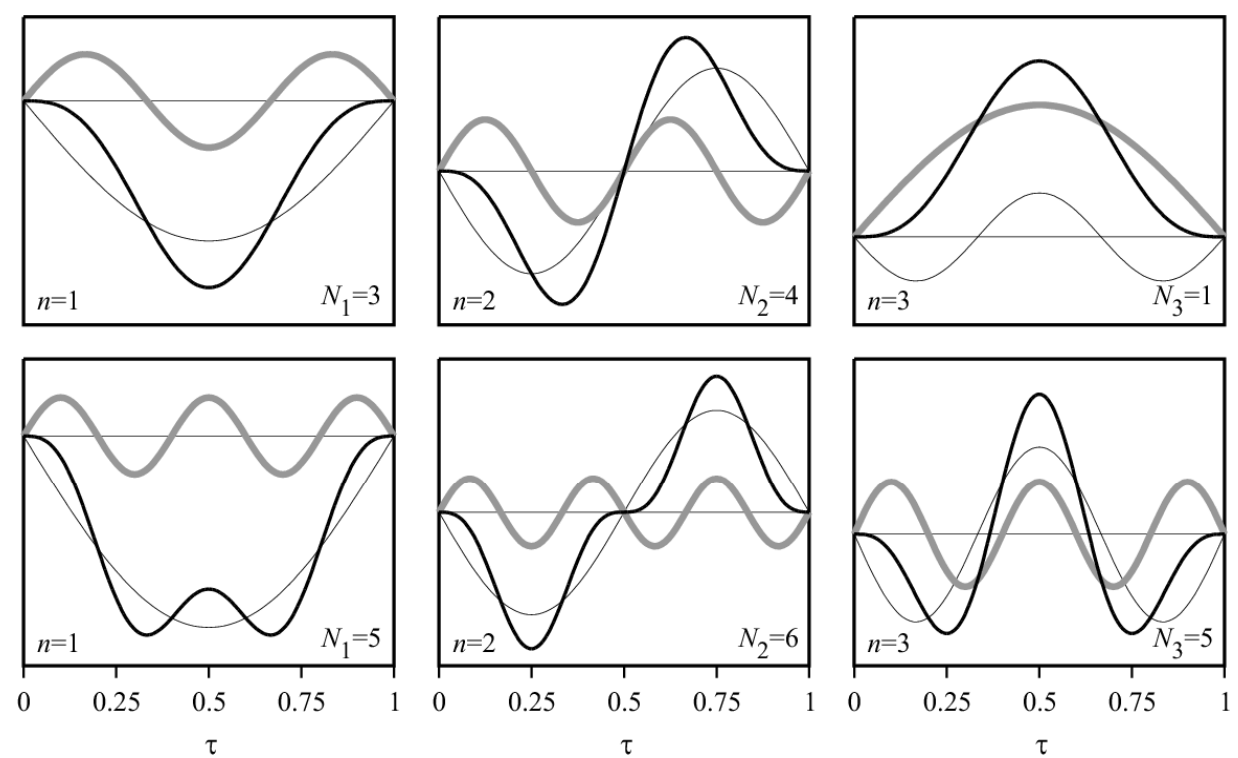

Fig. 6. Possible cancellations for different bending modes of an S-S beam. Homogeneous ( - ), particular $(-)$, and total $(-)$ vibration. 

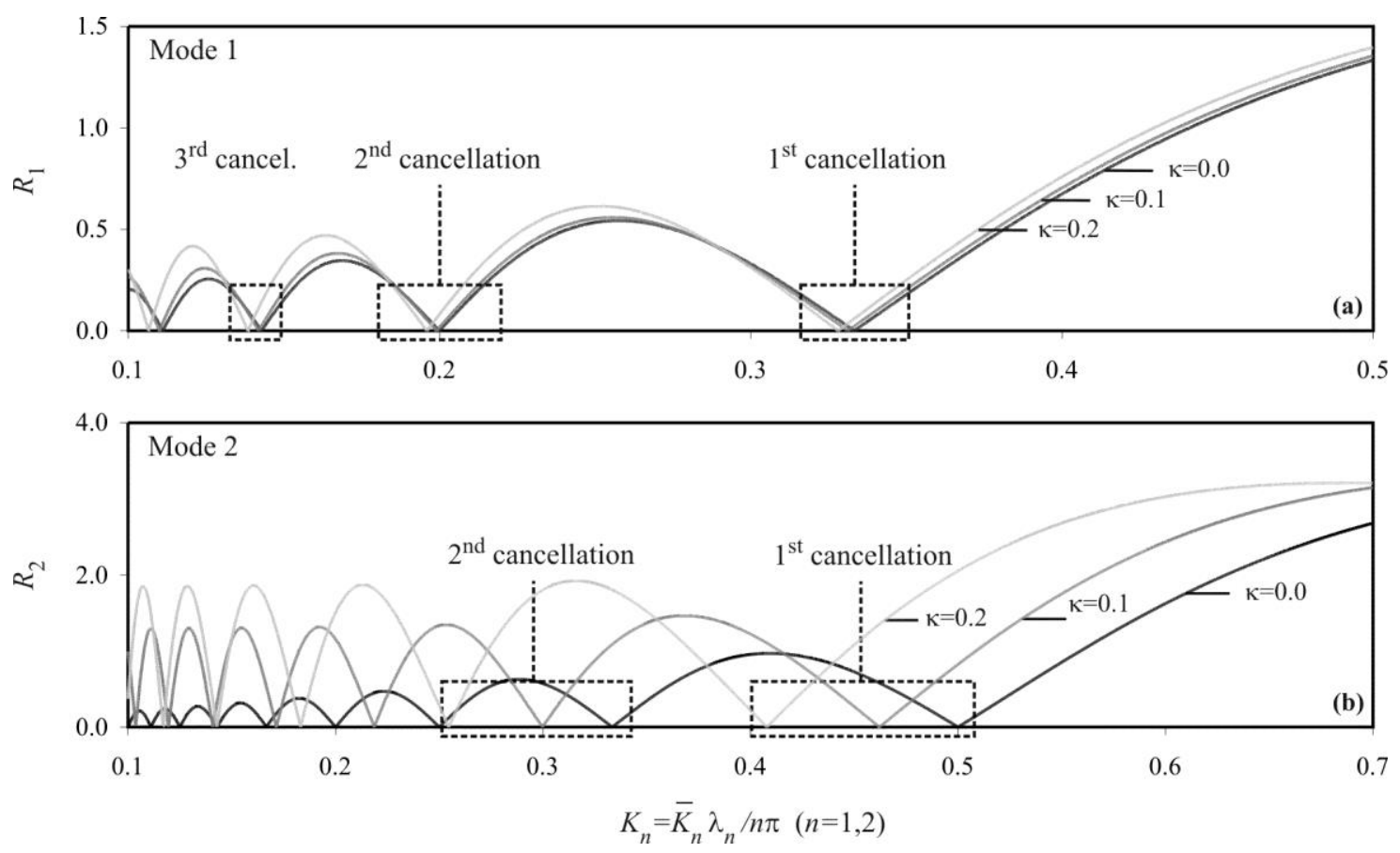

Fig. 7. (a) First and (b) second modal response amplitudes vs. speed parameter $K_{n}, n=1,2$ for values of $\kappa$ in the range $[0,0.2]$. 

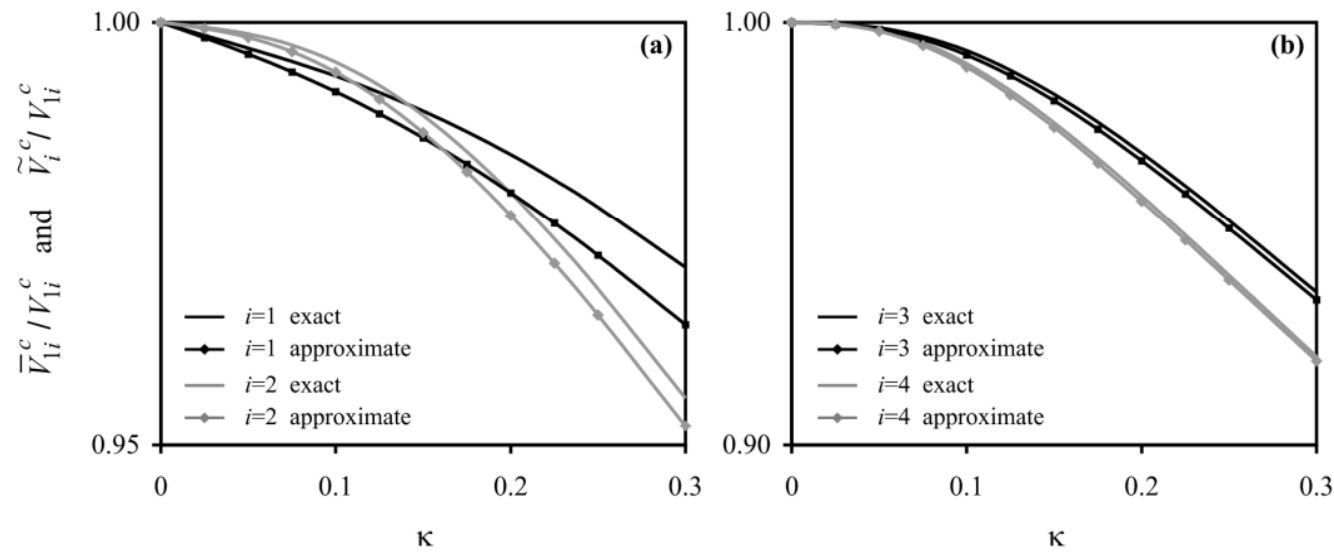

Fig. 8. Variation of the cancellation speeds with the supports flexibility. (a) First and second and (b) third and fourth cancellations of the fundamental mode. 


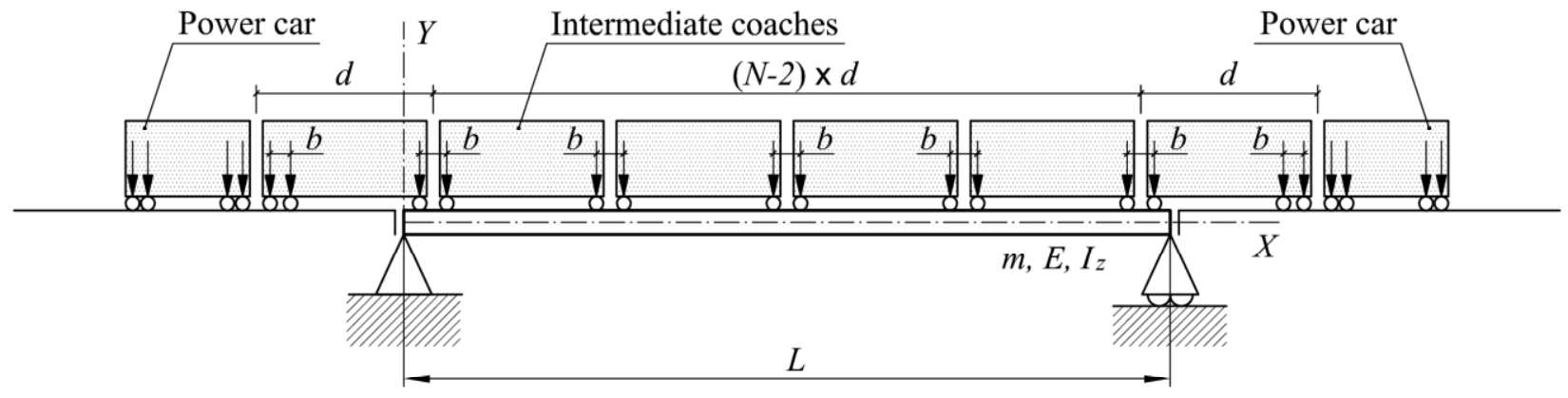

Fig. 9. Train composed of equidistant groups of loads. 


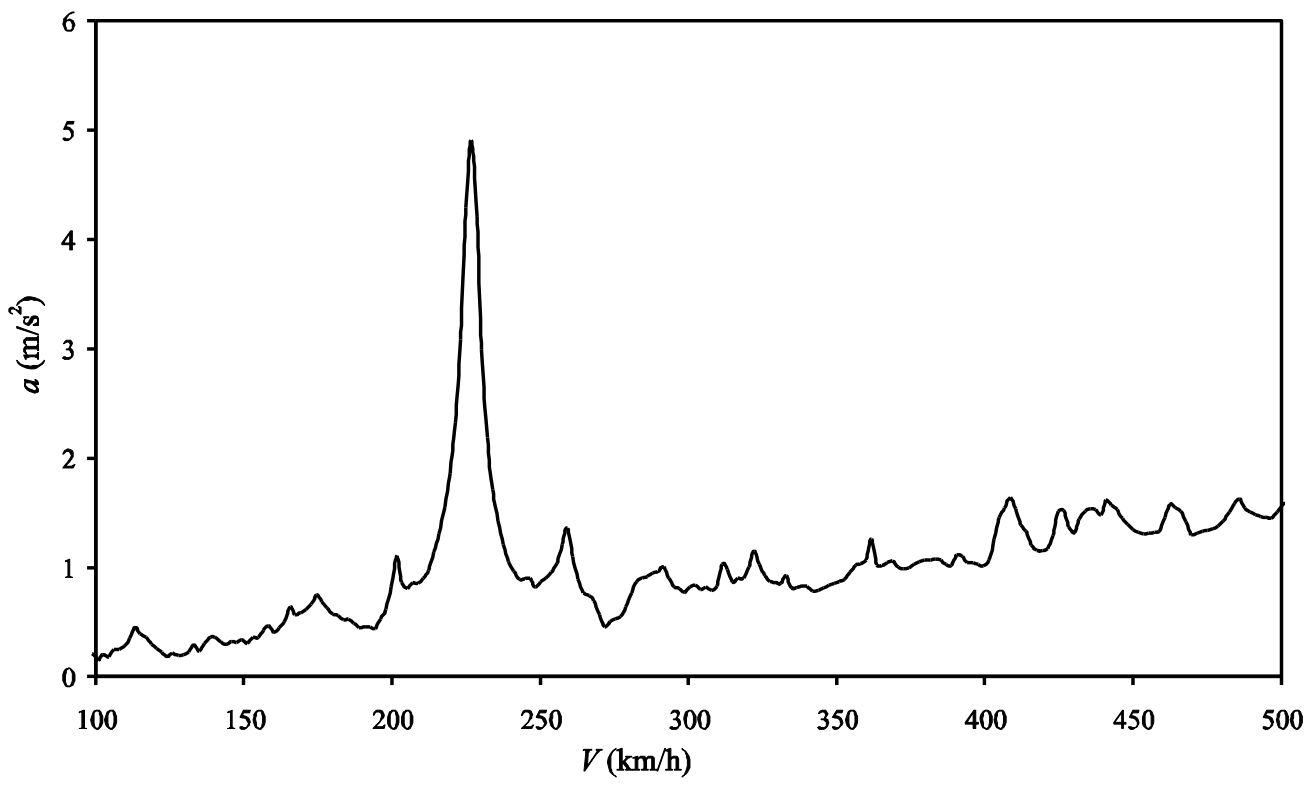

Fig. 10. Response of a $27 \mathrm{~m}$ bridge to A1 train from Eurocode $1(L / d=1.5)$. 


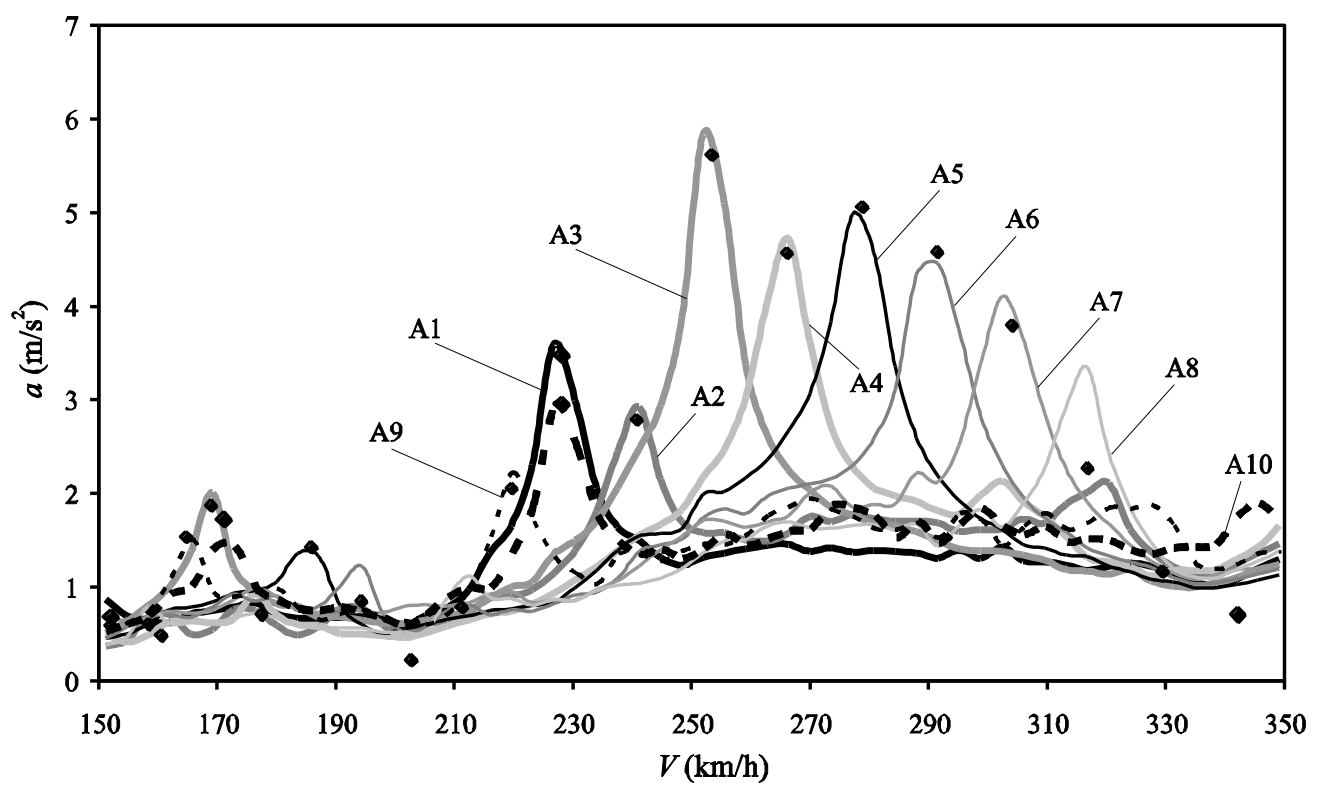

Fig. 11. Response of a $20 \mathrm{~m} \mathrm{~S}$-S bridge to the 10 trains of HSLM-A load model ( resonance values approximated with Eqs. (57)). 


\begin{tabular}{|c|c|c|c|c|c|c|c|c|}
\hline$\kappa$ & \multicolumn{4}{|c|}{$K_{1}^{m}$} & \multicolumn{4}{|c|}{$K_{11}^{c}$} \\
\hline $\mathbf{0}$ & 0.3860 & 0.2576 & 0.1687 & 0.1258 & 0.3333 & 0.2000 & 0.1429 & 0.1111 \\
\hline 0.1 & 0.3844 & 0.2556 & 0.1673 & 0.1245 & 0.3312 & 0.1991 & 0.1419 & 0.1100 \\
\hline 0.2 & 0.3845 & 0.2513 & 0.1634 & 0.1208 & 0.3282 & 0.1960 & 0.1385 & 0.1066 \\
\hline$\kappa$ & \multicolumn{4}{|c|}{$K_{2 i}^{m}$} & \multicolumn{4}{|c|}{$K_{2 i}^{c}$} \\
\hline 0 & 0.5570 & 0.4094 & 0.2886 & 0.2235 & 0.5000 & 0.3333 & 0.2500 & 0.2000 \\
\hline 0.1 & 0.5330 & 0.3682 & 0.2535 & 0.1924 & 0.4621 & 0.2998 & 0.2187 & 0.1715 \\
\hline 0.2 & 0.4919 & 0.3159 & 0.2132 & 0.1605 & 0.4079 & 0.2546 & 0.1832 & 0.1428 \\
\hline
\end{tabular}

Table 1. Values of $K_{n i}^{m}$ and $K_{n i}^{c}$ corresponding to attainable maxima of the free vibrations and cancellations for the first two modes of an E-S beam. 


\begin{tabular}{c|ccccc}
\hline Mode & $(-) i=1$ & $(+) i=1$ & $(+) i=2$ & $(+) i=3$ & $(+) i=4$ \\
\hline 1 & - & $1 / 3$ & $1 / 5$ & $1 / 7$ & $1 / 9$ \\
2 & - & $1 / 2$ & $1 / 3$ & $1 / 4$ & $1 / 5$ \\
3 & 3 & $3 / 5$ & $3 / 7$ & $1 / 3$ & $3 / 11$ \\
4 & 2 & $2 / 3$ & $1 / 2$ & $2 / 5$ & $1 / 3$ \\
\hline
\end{tabular}

Table 2. Values of $K_{n i}^{c}$ corresponding to cancellations of the first four modes (S-S beam). 


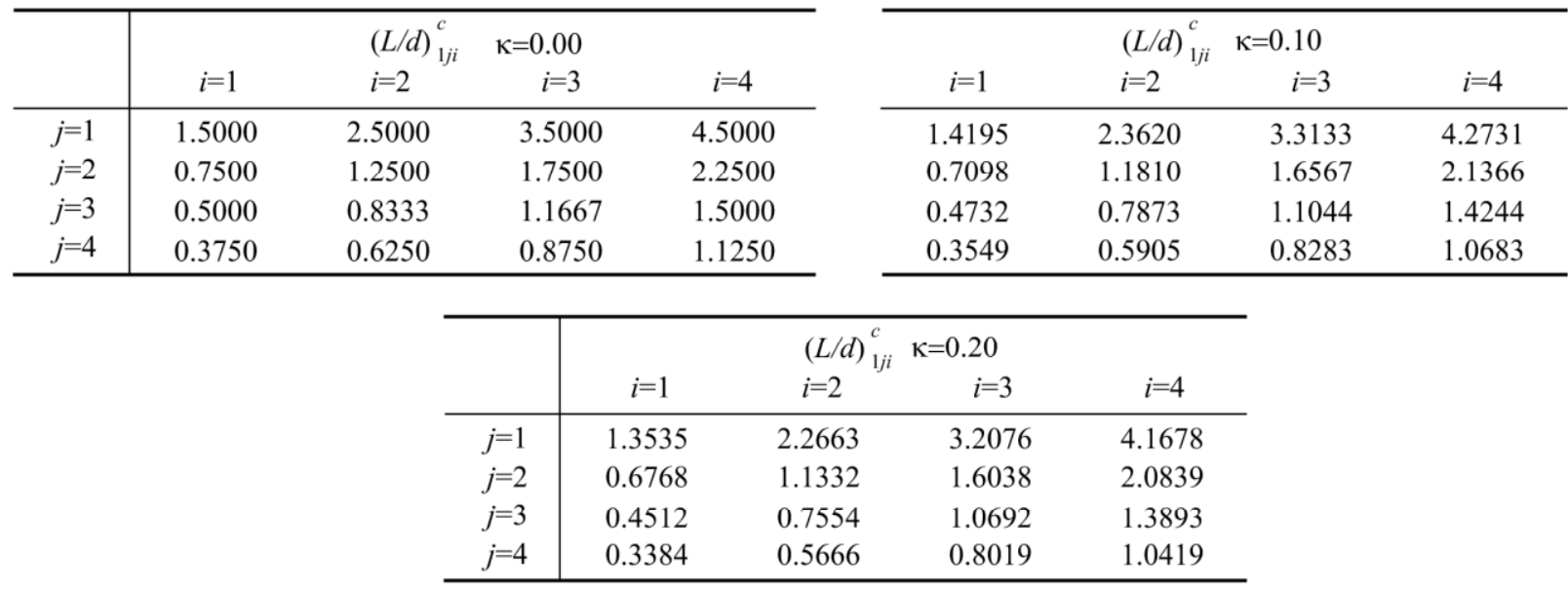

Table 3. Values of $L / d$ corresponding to cancellations of the first four resonances of the first bending mode for $\kappa=\{0.00,0.10,0.20\}$. 


\begin{tabular}{|c|c|c|c|c|c|c|c|c|c|}
\hline & $(\widehat{L / d})_{1 j}^{m}$ & $\begin{array}{l}(L / d)_{1 j i}^{m} \\
i=2\end{array}$ & \multicolumn{2}{|l|}{$\begin{array}{r}\kappa=0.00 \\
i=3\end{array}$} & $i=4$ & $\widehat{(L / d})_{1 j}^{m}$ & $\begin{array}{l}(L / d)^{m}{ }_{1 j i}^{m} \\
i=2\end{array}$ & $\begin{array}{r}\kappa=0.10 \\
i=3\end{array}$ & $i=4$ \\
\hline$j=1$ & 1.2953 & 1.9411 & \multicolumn{2}{|l|}{2.9640} & 3.9737 & 1.2233 & 1.8394 & 2.8101 & 3.7761 \\
\hline$j=2$ & 0.6477 & 0.9705 & \multicolumn{2}{|l|}{1.4820} & 1.9869 & 0.6117 & 0.9197 & 1.4051 & 1.8880 \\
\hline$j=3$ & 0.4318 & 0.6470 & \multicolumn{2}{|l|}{0.9880} & 1.3246 & 0.4078 & 0.6131 & 0.9367 & 1.2587 \\
\hline$j=4$ & 0.3238 & 0.4853 & \multicolumn{2}{|l|}{0.7410} & 0.9934 & 0.3058 & 0.4599 & 0.7025 & 0.9440 \\
\hline & & & \multicolumn{2}{|r|}{$(\widehat{L / d})_{1 j}^{m}$} & \multicolumn{2}{|c|}{$(L / d)_{1 i i}^{m} \kappa=0.20$} & $i=4$ & & \\
\hline & & & $j=1$ & 1.1550 & 1.7674 & 2.7180 & 3.6778 & & \\
\hline & & & $j=2$ & 0.5775 & 0.8837 & 1.3590 & 1.8389 & & \\
\hline & & & $j=3$ & 0.3850 & 0.5891 & 0.9060 & 1.2259 & & \\
\hline & & & $j=4$ & 0.2888 & 0.4418 & 0.6795 & 0.9195 & & \\
\hline
\end{tabular}

Table 4. Values of $L / d$ corresponding to locally maximum resonances and upper limits associated to overall maximum resonances of the first bending mode for $\kappa=\{0.00,0.10,0.20\}$. 


\begin{tabular}{|c|c|c|c|c|c|c|c|c|c|c|}
\hline$L(\mathrm{~m})$ & $f_{1}(\mathrm{~Hz})$ & $\zeta(\%)$ & $\begin{array}{c}a_{\max } \\
(5 \text { modes })\end{array}$ & Train & $\begin{array}{l}\text { Section } \\
(x / L)\end{array}$ & $\begin{array}{c}a_{\max } \\
(1 \text { mode })\end{array}$ & Train & $a_{\text {res }}$ & $\begin{array}{c}\text { error } \% \\
\text { ( } 5 \text { modes })\end{array}$ & $\begin{array}{c}\text { error } \% \\
(1 \text { mode })\end{array}$ \\
\hline & 8.000 & 1.0 & 82.2 & A10 & 0.50 & 80.6 & A10 & 87.5 & 6.4 & 8.6 \\
\hline \multirow[t]{3}{*}{10} & 12.464 & 2.0 & 24.1 & $\mathrm{~A} 1$ & 0.55 & 22.3 & A1 & 23.1 & -4.1 & 3.6 \\
\hline & 16.929 & 1.0 & 9.93 & $\mathrm{~A} 2$ & 0.60 & 9.63 & $\mathrm{~A} 2$ & 10.8 & 8.8 & 12.1 \\
\hline & 6.667 & 1.0 & 50.7 & $\mathrm{~A} 10$ & 0.50 & 50.7 & A10 & 55.0 & 8.5 & 8.5 \\
\hline \multirow[t]{3}{*}{12} & 10.719 & 1.0 & 23.6 & A3 & 0.50 & 23.1 & A3 & 24.5 & 3.8 & 6.1 \\
\hline & 14.771 & 2.0 & 4.30 & A3 & 0.55 & 4.25 & A3 & 4.11 & -4.4 & -3.3 \\
\hline & 5.714 & 1.0 & 68.4 & A3 & 0.50 & 68.9 & A3 & 67.1 & -1.9 & -2.6 \\
\hline \multirow[t]{3}{*}{14} & 9.438 & 1.0 & 20.1 & A7 & 0.55 & 19.8 & A7 & 21.6 & 7.5 & 9.1 \\
\hline & 13.162 & 2.0 & 4.02 & A6 & 0.40 & 3.71 & $\mathrm{~A} 6$ & 3.62 & -10.0 & -2.4 \\
\hline & 5.000 & 1.0 & 51.7 & A3 & 0.50 & 50.9 & A3 & 49.6 & -4.1 & -2.6 \\
\hline \multirow[t]{3}{*}{16} & 8.455 & 1.0 & 16.2 & $\mathrm{~A} 10$ & 0.50 & 15.9 & A10 & 17.0 & 4.9 & 6.9 \\
\hline & 11.911 & 2.0 & 4.29 & $\mathrm{~A} 1$ & 0.50 & 4.04 & A1 & 3.85 & -10.3 & -4.7 \\
\hline & 4.444 & 1.0 & 42.1 & A9 & 0.50 & 41.2 & A9 & 40.6 & -3.6 & -1.5 \\
\hline \multirow[t]{3}{*}{18} & 7.675 & 2.0 & 5.05 & $\mathrm{~A} 10$ & 0.45 & 4.56 & $\mathrm{~A} 10$ & 4.12 & -18.4 & -9.6 \\
\hline & 10.906 & 2.0 & 3.88 & $\mathrm{~A} 3$ & 0.50 & 3.70 & $\mathrm{~A} 3$ & 3.52 & -9.3 & -4.9 \\
\hline & 4.000 & 1.0 & 34.2 & A9 & 0.50 & 33.1 & A10 & 32.7 & -4.4 & -1.2 \\
\hline \multirow[t]{3}{*}{20} & 7.040 & 2.0 & 3.48 & $\mathrm{~A} 3$ & 0.50 & 3.17 & $\mathrm{~A} 3$ & 3.08 & -11.5 & -2.8 \\
\hline & 10.080 & 2.0 & 3.42 & $\mathrm{~A} 3$ & 0.50 & 3.26 & A3 & 3.08 & -9.9 & -5.5 \\
\hline & 3.783 & 2.0 & 19.2 & A10 & 0.50 & 18.9 & A10 & 18.5 & -3.6 & -2.1 \\
\hline \multirow[t]{3}{*}{22} & 6.585 & 2.0 & 2.95 & A6 & 0.45 & 2.86 & A7 & 2.76 & -6.4 & -3.5 \\
\hline & 9.386 & 2.0 & 3.00 & A6 & 0.45 & 2.85 & A7 & 2.76 & -8.0 & -3.2 \\
\hline & 3.593 & 2.0 & 14.7 & $\mathrm{~A} 10$ & 0.50 & 14.8 & $\overline{\mathrm{A} 10}$ & 14.6 & -0.7 & -1.4 \\
\hline \multirow[t]{3}{*}{24} & 6.194 & 1.0 & 6.03 & $\mathrm{~A} 1$ & 0.50 & 5.83 & A1 & 5.61 & -7.0 & -3.8 \\
\hline & 8.795 & 2.0 & 2.77 & A9 & 0.50 & 2.69 & A9 & 2.62 & -5.4 & -2.6 \\
\hline & 3.427 & 2.0 & 11.5 & $\mathrm{~A} 10$ & 0.50 & 11.4 & $\mathrm{~A} 10$ & 11.3 & -1.7 & -0.9 \\
\hline \multirow[t]{3}{*}{26} & 5.855 & 2.0 & 4.08 & $\mathrm{~A} 3$ & 0.50 & 3.98 & $\mathrm{~A} 3$ & 3.85 & -5.6 & -3.3 \\
\hline & 8.284 & 2.0 & 2.49 & A9 & 0.50 & 2.45 & A10 & 2.35 & -5.6 & -4.1 \\
\hline & 3.280 & 2.0 & 8.76 & A10 & 0.50 & 8.56 & A10 & 8.56 & -2.3 & 0.0 \\
\hline \multirow[t]{2}{*}{28} & 5.558 & 2.0 & 2.76 & A4 & 0.50 & 2.72 & $\mathrm{~A} 4$ & 2.70 & -2.2 & -0.7 \\
\hline & 7.837 & 2.0 & 1.96 & $\mathrm{~A} 10$ & 0.50 & 1.94 & $\mathrm{~A} 10$ & 1.84 & -6.1 & -5.2 \\
\hline \multirow{3}{*}{30} & 3.148 & 2.0 & 6.47 & A10 & 0.50 & 6.35 & $\mathrm{~A} 10$ & 6.31 & -2.5 & -0.6 \\
\hline & 5.296 & 1.0 & 2.92 & $\mathrm{~A} 1$ & 0.50 & 2.79 & $\mathrm{~A} 1$ & 2.69 & -7.9 & -3.6 \\
\hline & 7.443 & 2.0 & 1.27 & $\mathrm{~A} 10$ & 0.50 & 1.23 & $\mathrm{~A} 10$ & 1.17 & -7.9 & -4.9 \\
\hline
\end{tabular}

Table 5. Maximum vertical acceleration $a_{\max }\left(\mathrm{m} / \mathrm{s}^{2}\right)$ and comparison with $a_{\text {res }}$ obtained from Eqs. (57) for 33 test bridges. For each bridge $m(\mathrm{~kg} / \mathrm{m})=1000 \cdot L(\mathrm{~m})$. 